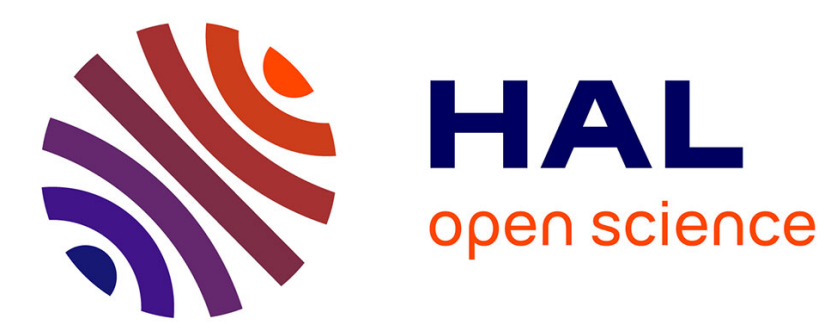

\title{
Strong Invariance Principles with Rate for "Reverse" Martingale Differences and Applications
}

Christophe Cuny, Florence Merlevède

\section{To cite this version:}

Christophe Cuny, Florence Merlevède. Strong Invariance Principles with Rate for "Reverse" Martingale Differences and Applications. Journal of Theoretical Probability, 2015, 28 (1), pp.137-183. 10.1007/s10959-013-0506-z . hal-00745647v2

\section{HAL Id: hal-00745647 \\ https://hal.science/hal-00745647v2}

Submitted on 13 Nov 2012

HAL is a multi-disciplinary open access archive for the deposit and dissemination of scientific research documents, whether they are published or not. The documents may come from teaching and research institutions in France or abroad, or from public or private research centers.
L'archive ouverte pluridisciplinaire HAL, est destinée au dépôt et à la diffusion de documents scientifiques de niveau recherche, publiés ou non, émanant des établissements d'enseignement et de recherche français ou étrangers, des laboratoires publics ou privés. 


\title{
Strong invariance principles with rate for "reverse" martingales and applications.
}

\author{
Christophe Cuny ${ }^{a}$ and Florence Merlevède ${ }^{b}$ \\ ${ }^{a}$ Laboratoire MAS, Ecole Centrale de Paris, Grande Voie des Vignes, 92295 Chatenay-Malabry cedex, \\ FRANCE. E-mail: christophe.cuny@ecp.fr \\ ${ }^{b}$ Université Paris Est, LAMA, CNRS UMR 8050, Bâtiment Copernic, 5 Boulevard Descartes, 77435 \\ Champs-Sur-Marne, FRANCE. E-mail: florence.merlevede@univ-mlv.fr
}

\begin{abstract}
In this paper, we obtain almost sure invariance principles with rate of order $n^{1 / p} \log ^{\beta} n, 2<p \leq 4$, for sums associated to a sequence of reverse martingale differences. Then, we apply those results to obtain similar conclusions in the context of some non-invertible dynamical systems. For instance we treat several classes of uniformly expanding maps of the interval (for possibly unbounded functions). A general result for $\phi$-dependent sequences is obtained in the course.
\end{abstract}

Mathematics Subject Classification (2010): 37E05, 37C30, 60F15.

Key words: Expanding maps, strong invariance principle, reverse martingale.

\section{Introduction}

Let $T$ be a map from $[0,1]$ to $[0,1]$ preserving a probability $\nu$ on $[0,1]$. Let $f$ be a measurable function such that $\nu(|f|)<\infty$. Define $S_{n}(f)=\sum_{i=0}^{n-1}\left(f \circ T^{i}-\nu(f)\right)$. According to the Birkhoff-Khintchine ergodic theorem, $n^{-1} S_{n}(f)$ satisfies the strong law of large numbers. One can go further in the study of the statistical properties of $S_{n}(f)$ and recently there have been extensive researches to study the almost sure invariance principle (ASIP) for $S_{n}(f)$. Roughly speaking, such a result ensures that the trajectories of a process can be matched with the trajectories of a Brownian motion in such a way that almost surely the error between the trajectories is negligible compared to the size of the trajectory. This result is more or less precise depending on the error term one obtains. In this paper, for some classes of uniformly expanding maps, we give conditions on $f$ ensuring that there exists a sequence of independent identically distributed (iid) Gaussian random variables $\left(Z_{i}\right)_{i \geq 1}$ such that

$$
\sup _{1 \leq k \leq n}\left|\sum_{i=0}^{k-1}\left(f \circ T^{i}-\nu(f)-Z_{i}\right)\right|=o\left(n^{1 / p} L(n)\right) \quad \text { almost surely, }
$$

for $p \in] 2,4]$ and $L$ an explicit slowly varying function. For different classes of piecewise expanding maps $T$ of $[0,1]$, almost sure invariance principles with good remainder estimates have been established by Melbourne and Nicol ([17], [18]) for Hölder observables, and by Merlevède and Rio [21] under rather mild integrability assumptions. For instance, for uniformly expanding maps as defined in Definition 3.1, Merlevède and Rio [21] obtained in (1.1) a rate of order $n^{1 / 3}(\log n)^{1 / 2}(\log \log n)^{(1+\varepsilon) / 3}$ for any $\varepsilon>0$ and a class of observables $f$ in $\mathbb{L}^{r}(\nu)$ where $r>3$. Because of the intrinsic time-orientation of the noninvertible dynamical systems studied in the papers above mentioned, the almost sure invariance principle cannot be obtained directly by mean of a martingale approximation as done for instance in [10]. In [21], the approximating Brownian motion is constructed with the help of the conditional quantile transform combined with a coupling method based, roughly speaking, on a conditional version of the KantorovitchRubinstein theorem. The approach used in [17] or in [18] when $d=1$ (see their appendix A) is based on the seminal paper by Philipp and Stout [24] which proves ASIP by combining martingale approximation with blocking arguments and the Strassen-Skorohod embedding. With this approach, they cannot reach better rates than $O\left(n^{\varepsilon+3 / 8}\right)$ in (1.1) for any $\varepsilon>0$ (see Remark 1.7 in [18]). Notice that recently Gouëzel [11] obtained a very general result concerning the rates in the ASIP for vector-valued observables of the iterates of dynamical systems by mean of spectral methods. In the particular case of expanding maps as defined in Definition 3.1, his result gives the rate $o\left(n^{1 / 4+\varepsilon}\right)$ for any $\varepsilon>0$ for some bounded vector-valued observables. 
On an other hand if we consider the strong approximation principle of the partial sums of real-valued functions of the Markov chain associated to the dynamical system, better rates can be reached. Let denote by $K$ the Perron-Frobenius operator of $T$ with respect to $\nu$. Recall that for any bounded measurable functions $f$ and $g$,

$$
\nu(f \cdot g \circ T)=\nu(K(f) g) .
$$

Let $\left(Y_{i}\right)_{i \geq 0}$ be a stationary Markov chain with invariant measure $\nu$ and transition Kernel $K$. The sequence $\left(Y_{i}\right)_{i \geq 0}$ corresponds to the iteration of the inverse branches of $T$. Combining a suitable martingale approximation with a sharp control of the approximation error in $\mathbb{L}^{p}$ for $p \geq 2$, and the SkorohodStrassen embedding theorem as done in Shao [26, Theorem 2.1], Dedecker, Merlevède and Doukhan [4] obtained projective conditions under which the strong approximation holds with an explicit rate, as in (1.1), for the partial sums associated to a stationary sequence of real valued random variables. In the Markov chain setting, the conditions involved in their corollaries 2.1 and 2.2 can be rewritten with the help of the transition kernel $K$. One can then wonder whether under the same conditions, the strong approximation principle also holds with the same rate for the partial sums associated to some observables of the iterates of the dynamical system. Indeed, it is well known (see for instance Lemma XI.3 in Hennion and Hervé [13]) that on the probability space $([0,1], \nu)$, the random vector $\left(T, T^{2}, \ldots, T^{n}\right)$ is distributed as $\left(Y_{n}, Y_{n-1}, \ldots, Y_{1}\right)$. Hence any information on the law of $\sum_{i=1}^{n}\left(f \circ T^{i}-\nu(f)\right)$ can be obtained by studying the law of $\sum_{i=1}^{n}\left(f\left(X_{i}\right)-\nu(f)\right)$. However, the reverse time property cannot be used to transfer directly the almost sure results for $\sum_{i=1}^{n}\left(f\left(Y_{i}\right)-\nu(f)\right)$ to the sum $\sum_{i=1}^{n}\left(f \circ T^{i}-\nu(f)\right)$. To prove results on the strong approximation principle for the partial sums of functions of the iterates of the dynamical system, it is then needed to work on the dynamical system itself. Therefore to prove that $\sum_{i=1}^{n}\left(f \circ T^{i}-\nu(f)\right)$ satisfies a strong approximation principle with the same rates than the one reached for the associated Markov chain, we shall approximate the partial sum associated to real-valued observables of the iterates of the dynamical system by a sum of reverse martingale differences $M_{n}^{*}$. To be more precise, we shall approximate $S_{n}(f)$ by $M_{n}^{*}=\sum_{k=1}^{n} d_{k}^{*}$ where $\left(d_{k}^{*}\right)_{k \in \mathbb{N}}$ is a sequence of real valued random variables that is measurable with respect to a non-increasing sequence of $\sigma$-algebras say $\left(\mathcal{G}_{k}\right)_{k \in \mathbb{N}}$ and such that $\mathbb{E}\left(d_{k}^{*} \mid \mathcal{G}_{k+1}\right)=0$ almost surely. An analogue of Theorem 2.1 in Shao [26] but in the context of the partial sums associated to a sequence of reverse martingale differences, is then needed. Up to our knowledge, this version for reverse martingale differences sequences does not exist in the literature. Starting from a reverse martingale version of the Skorohod-Strassen embedding theorem as done in Scott and Huggins [25], we shall prove it in Section 2 (see our Theorem 2.3).

Our paper is organized as follows. In Section 2, we state some results concerning the almost sure invariance principle with rates for sums associated to a sequence of reverse martingale differences. The proofs of these results are postponed to Section 6. In Section 3, we obtain rates of convergence in the ASIP for the sum of a large class of functions (non necessarily bounded) of the iterates of some classes of uniformly expanding maps, as well as for the partial sum associated to the corresponding Markov chain. A part of these results coming from a more general result on $\phi$-dependent sequences, we state and prove in Section 4 an ASIP with explicit rates for functions of a stationary sequence satisfying a mild $\phi$-dependent condition. Section 5 is devoted to the proofs of the results on expanding maps stated in Section 3 .

In this paper, we shall use sometimes the notation $a_{n} \ll b_{n}$ to mean that there exists a numerical constant $C$ not depending on $n$ such that $a_{n} \leq C b_{n}$, for all positive integers $n$.

\section{ASIP with rates for sums of differences of reverse martingales}

The next two results can be viewed as reverse martingales analogues to a result of Shao [26, Theorem 2.1]. The proof of the next result follows from the Skorohod embedding of reverse martingales in Brownian motion as obtained in Scott and Huggins [25] together with an estimate for Brownian motions given in Hanson and Russo [12, Theorem 3.2A]. The proofs of all the results presented in this section are postponed to Section 6 .

In this section we consider real random variables defined on a probability space $(\Omega, \mathcal{A}, \mathbb{P})$.

Proposition 2.1 Let $\left(\xi_{n}\right)_{n \in \mathbb{N}}$ be a sequence of square integrable variables adapted to a non-increasing filtration $\left(\mathcal{G}_{n}\right)_{n \in \mathbb{N}}$. Assume that $\mathbb{E}\left(\xi_{n} \mid \mathcal{G}_{n+1}\right)=0$ a.s. and $\delta_{n}^{2}:=\sum_{k \geq n} \mathbb{E}\left(\xi_{k}^{2}\right)<\infty$. Then $V_{n}^{2}:=$ $\sum_{k \geq n} \mathbb{E}\left(\xi_{k}^{2} \mid \mathcal{G}_{k+1}\right)$ is well defined a.s. and in $\mathbb{L}^{2}$ as well as $R_{n}=\sum_{k \geq n} \xi_{k}$. Let $\left(\alpha_{n}\right)_{n \in \mathbb{N}}$ be a sequence of 
non-increasing positive numbers with $\alpha_{n}=O\left(\delta_{n}^{2}\right)$ and $\alpha_{n} / \delta_{n}^{4} \rightarrow \infty$. Assume that

$$
\begin{gathered}
V_{n}^{2}-\delta_{n}^{2}=o\left(\alpha_{n}\right) \quad \mathbb{P} \text {-a.s. } \\
\sum_{n \geq 1} \alpha_{n}^{-\nu} \mathbb{E}\left(\left|\xi_{n}\right|^{2 \nu}\right) \quad \text { for some } 1 \leq \nu \leq 2 .
\end{gathered}
$$

Then, enlarging our probability space if necessary it is possible to find a standard Brownian motion $\left(B_{t}\right)_{t \geq 0}$, such that

$$
R_{n}-B_{\delta_{n}^{2}}=o\left(\left(\alpha_{n}\left(\left|\log \left(\delta_{n}^{2} / \alpha_{n}\right)\right|+\log \log \left(\alpha_{n}^{-1}\right)\right)\right)^{1 / 2}\right) \quad \mathbb{P} \text {-a.s. }
$$

Remark 2.2 It follows from the proof that if (2.1) holds with "big O" instead of "little o" then (2.3) holds with the same change.

Now, we give a result for partial sums associated to a sequence of reverse martingale differences rather than for tail series. It may be viewed as the analogue of Theorem 2.1 in Shao [26] but in the context of reverse martingale differences.

Theorem 2.3 Let $\left(X_{n}\right)_{n \in \mathbb{N}}$ be a sequence of square integrable random variables adapted to a nonincreasing filtration $\left(\mathcal{G}_{n}\right)_{n \in \mathbb{N}}$. Assume that $\mathbb{E}\left(X_{n} \mid \mathcal{G}_{n+1}\right)=0$ a.s., that $\sigma_{n}^{2}:=\sum_{k=1}^{n} \mathbb{E}\left(X_{k}^{2}\right) \rightarrow \infty$ and that $\sup _{n} \mathbb{E}\left(X_{n}^{2}\right)<\infty$. Let $\left(a_{n}\right)_{n \in \mathbb{N}}$ be a non-decreasing sequence of positive numbers such that $\left(a_{n} / \sigma_{n}^{2}\right)_{n \in \mathbb{N}}$ is non-increasing and $\left(a_{n} / \sigma_{n}\right)_{n \in \mathbb{N}}$ is non-decreasing. Assume that

$$
\begin{aligned}
& \sum_{k=1}^{n}\left(\mathbb{E}\left(X_{k}^{2} \mid \mathcal{G}_{k+1}\right)-\mathbb{E}\left(X_{k}^{2}\right)\right)=o\left(a_{n}\right) \quad \mathbb{P} \text {-a.s. } \\
& \sum_{n \geq 1} a_{n}^{-\nu} \mathbb{E}\left(\left|X_{n}\right|^{2 \nu}\right)<\infty \quad \text { for some } 1 \leq \nu \leq 2 .
\end{aligned}
$$

Then, enlarging our probability space if necessary it is possible to find a sequence $\left(Z_{k}\right)_{k \geq 1}$ of independent centered Gaussian variables with $\mathbb{E}\left(Z_{k}^{2}\right)=\mathbb{E}\left(X_{k}^{2}\right)$ such that

$$
\sup _{1 \leq k \leq n}\left|\sum_{i=1}^{k} X_{i}-\sum_{i=1}^{k} Z_{i}\right|=o\left(\left(a_{n}\left(\left|\log \left(\sigma_{n}^{2} / a_{n}\right)\right|+\log \log a_{n}\right)\right)^{1 / 2}\right) \quad \mathbb{P} \text {-a.s. }
$$

Remark 2.4 An inspection of the proof allows to weaken slightly some of the assumptions as follows. Assume that $\mathbb{E}\left(X_{n}^{2}\right)=O\left(\sigma_{n}^{2 s}\right)$ for some $0 \leq s<1$ instead of boundedness and assume that there exists $C>1$, such that for every $n \geq 1, \sup _{k \geq n}\left(a_{k} / \sigma_{k}^{2}\right) \leq C a_{n} / \sigma_{n}^{2}$, and $\inf _{k \geq n}\left(a_{k} / \sigma_{k}\right) \geq a_{n} /\left(C \sigma_{n}\right)$, instead of the corresponding monotonicity assumptions.

We derive now the functional LIL for the partial sums associated to a stationary and ergodic sequence of reverse martingale differences. In the next corollaries, we make use of $\theta: \Omega \mapsto \Omega$ a measurable transformation preserving the probability $\mathbb{P}$.

Corollary 2.5 Let $X_{0}$ in $\mathbb{L}^{2}$ and for $n \geq 1, X_{n}=X_{0} \circ \theta^{n}$. Assume that $\theta$ is ergodic. Let $\left(\mathcal{G}_{n}\right)_{n \geq 0}$ be a non-increasing filtration to which $\left(X_{n}\right)_{n \geq 0}$ is adapted and such that $\mathbb{E}\left(X_{n} \mid \mathcal{G}_{n+1}\right)=0$ a.s. Enlarging our probability space if necessary it is possible to find a sequence $\left(Z_{k}\right)_{k \geq 1}$ of independent centered Gaussian variables with $\mathbb{E}\left(Z_{k}^{2}\right)=\mathbb{E}\left(X_{1}^{2}\right)$ such that

$$
\sup _{1 \leq k \leq n}\left|\sum_{i=1}^{k} X_{i}-\sum_{i=1}^{k} Z_{i}\right|=o(\sqrt{n \log \log n}) \quad \text { P-a.s. }
$$

Remark 2.6 The Strassen functional law of the iterated logarithm (FLIL) follows from the corollary. Notice that a semi-FLIL has been proved by Wu [28].

We now give rate results in the strong invariance principle for the partial sums associated to a stationary sequence of reverse martingale differences. 
Corollary 2.7 Let $2<p<4$. Let $X_{0}$ be in $\mathbb{L}^{p}$ and for $n \geq 1, X_{n}=X_{0} \circ \theta^{n}$. Let $\left(\mathcal{G}_{n}\right)_{n \geq 0}$ be a nonincreasing filtration to which $\left(X_{n}\right)_{n \geq 0}$ is adapted and such that $\mathbb{E}\left(X_{n} \mid \mathcal{G}_{n+1}\right)=0$ a.s. Let $b(\cdot)$ be a positive non-decreasing slowly varying function, such that $x \mapsto x^{2 / p-1} b(x)$ is non-increasing. Assume that

$$
\sum_{k=1}^{n}\left(\mathbb{E}\left(X_{k}^{2} \mid \mathcal{G}_{k+1}\right)-\mathbb{E}\left(X_{k}^{2}\right)\right)=o\left(n^{2 / p} b(n)\right) \quad \mathbb{P} \text {-a.s. }
$$

Enlarging our probability space if necessary it is possible to find a sequence $\left(Z_{k}\right)_{k \geq 1}$ of independent centered Gaussian variables with $\mathbb{E}\left(Z_{k}^{2}\right)=\mathbb{E}\left(X_{1}^{2}\right)$ such that

$$
\sup _{1 \leq k \leq n}\left|\sum_{i=1}^{k} X_{i}-\sum_{i=1}^{k} Z_{i}\right|=o\left(n^{1 / p} \sqrt{b(n) \log n}\right) \quad \mathbb{P} \text {-a.s. }
$$

Corollary 2.8 Let $\mathcal{G}_{0}$ be a sub- $\sigma$-algebra of $\mathcal{A}$ satisfying $\theta^{-1}\left(\mathcal{G}_{0}\right) \subseteq \mathcal{G}_{0}$. Let $X_{0}$ be a $\mathcal{G}_{0}$-measurable random variable in $\mathbb{L}^{4}$. For $n \geq 1$, let $X_{n}=X_{0} \circ \theta^{n}$ and $\mathcal{G}_{n}=\theta^{-n}\left(\mathcal{G}_{0}\right)$. Assume that $\theta$ is ergodic and that $\mathbb{E}\left(X_{n} \mid \mathcal{G}_{n+1}\right)=0$ a.s. Assume that

$$
\sum_{k=1}^{n}\left(\mathbb{E}\left(X_{k}^{2} \mid \mathcal{G}_{k+1}\right)-\mathbb{E}\left(X_{k}^{2}\right)\right)=O\left((n \log \log n)^{1 / 2}\right) \quad \mathbb{P} \text {-a.s. }
$$

Enlarging our probability space if necessary it is possible to find a sequence $\left(Z_{k}\right)_{k \geq 1}$ of independent centered Gaussian variables with $\mathbb{E}\left(Z_{k}^{2}\right)=\mathbb{E}\left(X_{1}^{2}\right)$ such that

$$
\sup _{1 \leq k \leq n}\left|\sum_{i=1}^{k} X_{i}-\sum_{i=1}^{k} Z_{i}\right|=O\left(n^{1 / 4}(\log n)^{1 / 2}(\log \log n)^{1 / 4}\right) \quad \text { P-a.s. }
$$

Remark 2.9 If we consider usual martingale differences, so more precisely if $\left(X_{n}\right)_{n \in \mathbb{N}}$ is a stationary sequence in $\mathbb{L}^{p}$ for a real $\left.\left.p \in\right] 2,4\right]$ adapted to a non-decreasing filtration $\left(\mathcal{F}_{n}\right)_{n \in \mathbb{N}}$ (assume moreover that $\mathcal{F}_{n}=\theta^{-n} \mathcal{F}_{0}$ when $\left.p=4\right)$ and such that $\mathbb{E}\left(X_{n} \mid \mathcal{F}_{n-1}\right)=0$ a.s., the usual Skorohod-Strassen embedding theorem gives similar results to Corollaries 2.7 and 2.8. Indeed starting from Theorem 2.1 in Shao [26] when $p \in] 2,4[$ or from its proof when $p=4$ (notice that by stationarity -of the process and the filtration-, the stopping times used to construct the approximating Brownian motion can be chosen to be stationary), and using the arguments developed to get Corollaries 2.7 and 2.8, we infer that if there exists a positive non-decreasing slowly varying function $b(\cdot)$, such that

$$
\left.\left.\sum_{k=1}^{n}\left(\mathbb{E}\left(X_{k}^{2} \mid \mathcal{F}_{k-1}\right)-\mathbb{E}\left(X_{k}^{2}\right)\right)=o\left(n^{2 / p} b(n)\right) \quad \mathbb{P} \text {-a.s. when } p \in\right] 2,4\right],
$$

or if, $\left(X_{n}\right)_{n \in \mathbb{N}}$ is ergodic and

$$
\sum_{k=1}^{n}\left(\mathbb{E}\left(X_{k}^{2} \mid \mathcal{F}_{k-1}\right)-\mathbb{E}\left(X_{k}^{2}\right)\right)=O\left((n \log \log n)^{1 / 2}\right) \quad \mathbb{P} \text {-a.s. when } p=4
$$

then, enlarging our probability space if necessary it is possible to find a sequence $\left(Z_{k}\right)_{k \geq 1}$ of independent centered Gaussian variables with $\mathbb{E}\left(Z_{k}^{2}\right)=\mathbb{E}\left(X_{1}^{2}\right)$ such that the rates of approximation in the strong invariance principle are the same as those given by (2.9) and (2.10).

\section{$3 \quad$ ASIP with rates for uniformly expanding maps}

Several classes of uniformly expanding maps of the interval are considered in the literature. In Theorem 3.4 below, we shall consider the following definition:

Definition 3.1 A map $T:[0,1] \rightarrow[0,1]$ is uniformly expanding if it belongs to the class $\mathcal{C}$ defined in Broise [1, Section 2.1 page 11] and if

1. There is a unique absolutely continuous invariant probability measure $\nu$, whose density $h$ is such that $\frac{1}{h} \mathbf{1}_{h>0}$ has bounded variation. 
2. The system $(T, \nu)$ is mixing in the ergodic theoretic sense.

We refer also to Definition 1.1 in [6] for a more complete description. Some well known examples of maps satisfying the above conditions are:

1. $T(x)=\beta x-[\beta x]$ for $\beta>1$. These maps are called $\beta$-transformations.

2. $I$ is the finite union of disjoint intervals $\left(I_{k}\right)_{1 \leq k \leq n}$, and $T(x)=a_{k} x+b_{k}$ on $I_{k}$, with $\left|a_{k}\right|>1$.

3. $T(x)=a\left(x^{-1}-1\right)-\left[a\left(x^{-1}-1\right)\right]$ for some $a>0$. For $a=1$, this transformation is known as the Gauss map.

Our aim is to obtain explicit rates in the strong invariance principle for $\sum_{i=0}^{n-1}\left(f \circ T^{i}-\nu(f)\right)$ and $\sum_{i=1}^{n}\left(f\left(Y_{i}\right)-\nu(f)\right)\left(\left(Y_{i}\right)_{i \geq 0}\right.$ corresponding to the iterations of the inverse branches of $\left.T\right)$ when $T$ is a dynamical system defined in Definition 3.1, and $f$ belongs to a large class of functions non necessarily bounded. Such classes are described in the following definition.

Definition 3.2 If $\mu$ is a probability measure on $\mathbb{R}$ and $p \in[2, \infty), M \in(0, \infty)$, let $\operatorname{Mon}_{p}(M, \mu)$ denote the set of functions $f: \mathbb{R} \rightarrow \mathbb{R}$ which are monotonic on some interval and null elsewhere and such that $\mu\left(|f|^{p}\right) \leq M^{p}$. Let $\operatorname{Mon}_{p}^{c}(M, \mu)$ be the closure in $\mathbb{L}^{p}(\mu)$ of the set of functions which can be written as $\sum_{\ell=1}^{L} a_{\ell} f_{\ell}$, where $\sum_{\ell=1}^{L}\left|a_{\ell}\right| \leq 1$ and $f_{\ell} \in \operatorname{Mon}_{p}(M, \mu)$.

Remark 3.3 In previous papers, see for instance [6], the closure in $\mathbb{L}^{1}(\mu)$ was used in the definition above. It turns out that both definitions coincide. Indeed, a sequence bounded in $\mathbb{L}^{p}(\mu)$ and converging in $\mathbb{L}^{1}(\mu)$, converges for the weak topology in $\mathbb{L}^{p}(\mu)$. To conclude, recall that, by the Hahn-Banach theorem, in any Banach space, the weak closure of a convex set is equal to its strong closure.

Our main theorem follows. For uniformly expanding maps as defined in Definition 3.1, it involves an $\mathbb{L}^{p}$-integrability condition of the observables.

Theorem 3.4 Let $T$ be a uniformly expanding map as defined in Definition 3.1, with absolutely continuous invariant measure $\nu$. Let $p \in] 2,4]$. Then, for any $M>0$ and any $f \in \operatorname{Mon}_{p}^{c}(M, \nu)$, the series

$$
\sigma^{2}=\sigma^{2}(f)=\nu\left((f-\nu(f))^{2}\right)+2 \sum_{k>0} \nu\left((f-\nu(f)) f \circ T^{k}\right)
$$

converges absolutely to some non negative number.

1. Let $\left(Y_{i}\right)_{i \geq 1}$ be a stationary Markov chain with transition kernel $K$ defined by (1.2) and invariant measure $\nu$. Enlarging the underlying probability space if necessary, there exists a sequence $\left(Z_{i}\right)_{i \geq 0}$ of iid Gaussian random variables with mean zero and variance $\sigma^{2}$ defined by (3.1), such that

$$
\left.\sup _{1 \leq k \leq n} \mid \sum_{i=1}^{k}\left(f\left(Y_{i}\right)-\nu(f)\right)-Z_{i}\right) \mid= \begin{cases}o\left(n^{1 / p}(\log n)^{1-2 / p}\right) \text { a.s. } & \text { if } p \in] 2,4[ \\ O\left(n^{1 / 4}(\log n)^{1 / 2}(\log \log n)^{1 / 4}\right) \text { a.s. } & \text { if } p=4 .\end{cases}
$$

2. Enlarging the probability space $([0,1], \nu)$ if necessary, there exists a sequence $\left(Z_{i}^{*}\right)_{i \geq 0}$ of iid Gaussian random variables with mean zero and variance $\sigma^{2}$ defined by (3.1), such that

$$
\left.\sup _{1 \leq k \leq n} \mid \sum_{i=0}^{k-1}\left(f \circ T^{i}-\nu(f)\right)-Z_{i}^{*}\right) \mid= \begin{cases}o\left(n^{1 / p}(\log n)^{1-2 / p}\right) \text { a.s. } & \text { if } p \in] 2,4[ \\ O\left(n^{1 / 4}(\log n)^{1 / 2}(\log \log n)^{1 / 4}\right) \text { a.s. } & \text { if } p=4 .\end{cases}
$$

As we shall see in the proof of Theorem 3.4, Item 1 will follow from a more general result on $\phi$-dependent sequences. This general result is presented in a separate section (see Section 4) since it has an interest in itself. Item 2 is obtained by considering an approximation by partial sums associated to a sequence (which is non stationary when $p \in] 2,4[$ ) of reverse martingale differences and by applying the results of Section 2. As we shall see, the reverse time property mentioned in the introduction allows to make links with estimates obtained to prove Item 1 . Notice that when $p=2$, the strong invariance principle (therefore with the rate $\left.o\left(n^{1 / 2}(\log \log n)^{1 / 2}\right)\right)$ has been proved recently in [6] (see Item 3 of their Theorem 1.5). Notice that the proof of the strong invariance principle obtained in [6] combines different approximation arguments. The observable is approximated by a function with better integrability properties, the almost 
sure invariance principle with rates obtained in [21] is then used for this new function, and a bounded law of the iterated logarithm is proved to make it possible to pass the results from the better function to the original function. Let us emphasize that the strong invariance principle obtained in [6] could be also proved by using a direct approximation by partial sums associated to a sequence of reverse martingale differences as we do in the proof of Theorem 3.4 above, and by using our Corollary 2.5.

Let us mention that when $p=4$, Theorem 3.4 is a consequence of the following more general result.

Theorem 3.5 Let $T$ be a map from $[0,1]$ to $[0,1]$ preserving a probability $\nu$ on $[0,1]$, and let $K$ be defined by (1.2). Let $f$ be a measurable function such that $\nu\left(f^{4}\right)<\infty$. Assume that there exists $\left.\left.\gamma \in\right] 0,1\right]$ such that

$$
\sum_{n>0}(\log n)^{3} n^{\frac{1}{\gamma}+\frac{1}{2}}\left\|K^{n}(f)-\nu(f)\right\|_{4, \nu}^{2}<\infty
$$

and

$$
\sum_{n>0}(\log n)^{3} n^{2 \gamma} \sup _{i \geq j \geq n}\left\|K^{j}\left(f K^{i-j}(f)\right)-\nu\left(f K^{i-j}(f)\right)\right\|_{2, \nu}^{2}<\infty .
$$

Then, the series $\sigma^{2}$ defined by (3.1) converges absolutely and both items of Theorem 3.4 holds with rate $O\left(n^{1 / 4}(\log n)^{1 / 2}(\log \log n)^{1 / 4}\right)$.

Remark 3.6 We would like to emphasize that the strategy of proof of Item 2 for both Theorems 3.4 and 3.5 is to approximate $S_{n}(f)=\sum_{i=0}^{n-1}\left(f \circ T^{i}-\nu(f)\right)$ by a partial sum associated to a sequence of reverse martingale differences (possibly non stationary), let say $M_{n}^{*}=\sum_{i=0}^{n-1} d_{i}^{*}$, to apply our strong approximation results given in Section 2 and to have a nice control of the approximation error between $S_{n}(f)$ and $M_{n}^{*}$. A careful analysis of the proof of Theorem 2.4 and its Corollary 2.7 in [4] together with the arguments developed in the proof of Item 2 of our Theorems 3.4 and 3.5, show that our strategy of proof also gives new results when we consider classes of expanding maps with a neutral fixed point at zero such as the generalized Pomeau-Manneville (GPM) map as defined in Definition 1.1 of [5]. Therefore we infer that if $T$ is a GPM map with parameter $\gamma \in(0,1)$ and $f$ is a function satisfying Condition (3.22) in [4], then the conclusion of Corollary 3.18 in [4] also holds for $S_{n}(f)$. In particular, if $f$ is a bounded variation function and $T$ is a GPM map with parameter $\gamma \in\left(0, \delta^{-1}\right]$ where $\delta=p+1-2 / p$ and $\left.\left.p \in\right] 2,4\right]$, then $S_{n}(f)$ satisfies an almost sure invariance principle with rate o $\left(n^{1 / p} \log n\right)$.

For a map $T$ from $[0,1]$ to $[0,1]$ preserving a probability $\nu$ on $[0,1]$ and $\left(Y_{i}\right)_{i \geq 0}$ its associated Markov chain with invariant measure $\nu$ and transition Kernel $K$ defined by (1.2), Theorem 3.5 also allows to obtain rates in the strong invariance principle for $\sum_{i=1}^{n}\left(f \circ T^{i}-\nu(f)\right)$ (or for the partial sum of its associated Markov chain) when $f$ has a modulus of continuity that is dominated by a concave and non-decreasing function and the condition (3.4) below is satisfied.

For any integer $k$, we denote by $Q_{k}$ the operator defined as follows: $\mathbb{E}\left(g\left(Y_{0}, Y_{k}\right) \mid Y_{0}=x\right)=Q_{k}(g)(x)$. Let $\Lambda_{1}(\mathbb{R})$ be the set of functions from $\mathbb{R}$ to $\mathbb{R}$ that are 1-Lipschitz and let $\Lambda_{1}\left(\mathbb{R}^{2}\right)$ be the set of functions $h$ from $\mathbb{R}^{2}$ to $\mathbb{R}$ such that

$$
\left|h\left(x_{1}, y_{1}\right)-h\left(x_{2}, y_{2}\right)\right| \leq \frac{1}{2}\left|x_{1}-x_{2}\right|+\frac{1}{2}\left|y_{1}-y_{2}\right|
$$

Denote by $\|\cdot\|_{\infty, \mu}$ the essential supremum norm with respect to $\nu$. Assume that there exist $C>0$ and $\rho \in] 0,1\left[\right.$ such that for any $(i, j) \in \mathbb{N}^{2}$,

$$
\sup _{g \in \Lambda_{1}(\mathbb{R})}\left\|K^{i}(g)-\nu(g)\right\|_{\infty, \nu} \leq C \rho^{i} \text { and } \sup _{j \geq 0} \sup _{h \in \Lambda_{1}\left(\mathbb{R}^{2}\right)}\left\|K^{i} \circ Q_{j}(h)-\nu\left(Q_{j}(h)\right)\right\|_{\infty, \nu} \leq C \rho^{i} .
$$

Theorem 3.7 Let $T$ be a map from $[0,1]$ to $[0,1]$ preserving a probability $\nu$ on $[0,1]$ and let $\left(Y_{i}\right)_{i \geq 0}$ be a stationary Markov chain with invariant measure $\nu$ and transition Kernel $K$ defined by (1.2). Assume that condition (3.4) is satisfied. Let $f$ from $\mathbb{R}$ to $\mathbb{R}$ such that $|f(x)-f(y)| \leq c(|x-y|)$, for some concave and non-decreasing function c satisfying

$$
\int_{0}^{1}|\log t|^{2 / \sqrt{3}}\left|\log \log \left(2 t^{-1}\right)\right|^{3} \frac{c(t)}{t} d t<\infty .
$$

Then the conclusion of Theorem 3.5 holds. 
Note that (3.5) holds if $c(t) \leq D|\log (t)|^{-\gamma}$ for some $D>0$ and some $\gamma>1+2 / \sqrt{3}$. Therefore Theorem 3.7 applies to the functions from $[0,1]$ to $\mathbb{R}$ which are $\alpha$-Hölder for some $\alpha \in] 0,1]$.

Dedecker and Prieur [9, Section 7.2, Example 4.4] have shown that condition (3.4) is satisfied for a large class of uniformly expanding maps such as those considered in [2]. The conditions imposed on the class of the expanding maps they consider are slightly more restrictive than those considered in Definition 3.1. In particular they are defined by mean of finite partitions of $[0,1]$.

\section{A general ASIP result for a class of weakly dependent se- quences}

Let $(\Omega, \mathcal{A}, \mathbb{P})$ be a probability space, and let $\theta: \Omega \mapsto \Omega$ be a bijective bimeasurable transformation preserving the probability $\mathbb{P}$. Let $\mathcal{F}_{0}$ be a sub- $\sigma$-algebra of $\mathcal{A}$ satisfying $\mathcal{F}_{0} \subseteq \theta^{-1}\left(\mathcal{F}_{0}\right)$. Define the nondecreasing filtration $\left(\mathcal{F}_{i}\right)_{i \in \mathbb{Z}}$ by $\mathcal{F}_{i}=\theta^{-i}\left(\mathcal{F}_{0}\right)$. We shall sometimes denote by $\mathbb{E}_{i}$ the conditional expectation with respect to $\mathcal{F}_{i}$, and we shall set $P_{i}(\cdot)=\mathbb{E}_{i}(\cdot)-\mathbb{E}_{i-1}(\cdot)$.

Definition 4.1 For any integrable random variable $X$, let us write $X^{(0)}=X-\mathbb{E}(X)$. For any random variable $Y=\left(Y_{1}, \cdots, Y_{k}\right)$ with values in $\mathbb{R}^{k}$ and any $\sigma$-algebra $\mathcal{F}$, let

$$
\phi(\mathcal{F}, Y)=\sup _{\left(x_{1}, \ldots, x_{k}\right) \in \mathbb{R}^{k}}\left\|\mathbb{E}\left(\prod_{j=1}^{k}\left(\mathbf{1}_{Y_{j} \leq x_{j}}\right)^{(0)} \mid \mathcal{F}\right)^{(0)}\right\|_{\infty} .
$$

For a sequence $\mathbf{Y}=\left(Y_{i}\right)_{i \in \mathbb{Z}}$, where $Y_{i}=Y_{0} \circ \theta^{i}$ and $Y_{0}$ is an $\mathcal{F}_{0}$-measurable and real-valued random variable, let

$$
\phi_{k, \mathbf{Y}}(n)=\max _{1 \leq l \leq k} \sup _{n \leq i_{1} \leq \ldots \leq i_{l}} \phi\left(\mathcal{F}_{0},\left(Y_{i_{1}}, \ldots, Y_{i_{l}}\right)\right) .
$$

Theorem 4.2 Let $X_{i}=f\left(Y_{i}\right)-\mathbb{E}\left(f\left(Y_{i}\right)\right)$, where $Y_{i}=Y_{0} \circ \theta^{i}$ and $Y_{0}$ is an $\mathcal{F}_{0}$-measurable random variable. Let $P_{Y_{0}}$ be the distribution of $Y_{0}$ and $\left.\left.p \in\right] 2,4\right]$. Assume that $f$ belongs to $\operatorname{Mon}_{p}^{c}\left(M, P_{Y_{0}}\right)$ for some $M>0$ and that

$$
\left.\sum_{k \geq 1} k^{1 / \sqrt{3}-1 / 2} \phi_{2, \mathbf{Y}}^{1 / 2}(k)<\infty \quad \text { if } p \in\right] 2,4\left[\text { and } \sum_{k \geq 2}(\log k)^{3} k^{2 / \sqrt{3}} \phi_{2, \mathbf{Y}}(k)<\infty \text { if } p=4\right. \text {. }
$$

Then, enlarging our probability space if necessary, there exists a sequence $\left(Z_{i}\right)_{i \geq 0}$ of iid Gaussian random variables with mean zero and variance $\sigma^{2}$ defined by the absolutely converging series

$$
\sigma^{2}=\sum_{k \in \mathbb{Z}} \operatorname{Cov}\left(X_{0}, X_{k}\right)
$$

such that

$$
\sup _{1 \leq k \leq n}\left|\sum_{i=1}^{k}\left(X_{i}-Z_{i}\right)\right|= \begin{cases}o\left(n^{1 / p}(\log n)^{1-2 / p}\right) \text { a.s. } & \text { if } p \in] 2,4[ \\ O\left(n^{1 / 4}(\log n)^{1 / 2}(\log \log n)^{1 / 4}\right) \text { a.s. } & \text { if } p=4 .\end{cases}
$$

Notice that an application of Corollary 2.1 in [4] would give a rate of convergence of order $o\left(n^{1 / p}(\log n)^{(t+1) / 2}\right)$ with $t>2 / p$. As we shall see in the proof of the above theorem, the improvement of the power in the logarithmic term is achieved via some truncation arguments.

The proof makes use of the following lemma (see Lemma 5.2 in Dedecker, Gouëzel and Merlevède [6]).

Lemma 4.3 Let $\mathbf{Y}=\left(Y_{i}\right)_{i \in \mathbb{Z}}$, where $Y_{i}=Y_{0} \circ \theta^{i}$ and $Y_{0}$ is an $\mathcal{F}_{0}$-measurable random variable. Let $f$ and $g$ be two functions from $\mathbb{R}$ to $\mathbb{R}$ which are monotonic on some interval and null elsewhere. Let $p \in[1, \infty]$. If $\left\|f\left(Y_{0}\right)\right\|_{p}<\infty$, then, for any positive integer $k$,

$$
\left\|\mathbb{E}\left(f\left(Y_{k}\right) \mid \mathcal{F}_{0}\right)-\mathbb{E}\left(f\left(Y_{k}\right)\right)\right\|_{p} \leq 2\left(2 \phi_{1, \mathbf{Y}}(k)\right)^{(p-1) / p}\left\|f\left(Y_{0}\right)\right\|_{p} .
$$

If moreover $p \geq 2$ and $\left\|g\left(Y_{0}\right)\right\|_{p}<\infty$, then for any positive integers $i \geq j \geq k$,

$$
\left\|\mathbb{E}\left(f\left(Y_{i}\right)^{(0)} g\left(Y_{j}\right)^{(0)} \mid \mathcal{F}_{0}\right)-\mathbb{E}\left(f\left(Y_{i}\right)^{(0)} g\left(Y_{j}\right)^{(0)}\right)\right\|_{p / 2} \leq 8\left(4 \phi_{2, \mathbf{Y}}(k)\right)^{(p-2) / p}\left\|f\left(Y_{0}\right)\right\|_{p}\left\|g\left(Y_{0}\right)\right\|_{p} .
$$




\subsection{Proof of Theorem 4.2 for $2<p<4$}

Let $f \in \operatorname{Mon}_{p}^{c}\left(M, P_{Y_{0}}\right)$. We shall first prove that $\sum_{\ell \geq 0}\left\|\mathbb{E}_{0}\left(X_{\ell}\right)\right\|_{p}<\infty$ provided that $\sum_{k \geq 1} \phi_{1, \mathbf{Y}}^{(p-1) / p}(k)<$ $\infty$ (notice that this condition is implied by (4.1)). This will entail that $d_{0}:=\sum_{\ell>0} P_{0}\left(X_{\ell}\right)$ and $r_{0}:=\sum_{\ell \geq 1} \mathbb{E}_{0}\left(X_{\ell}\right)$ are well defined and that we have the martingale-coboundary decomposition $X_{0}=$ $d_{0}+r_{0} \circ \theta^{-1}-r_{0}$. Then the series $\sigma^{2}=\sum_{k \in \mathbb{Z}} \operatorname{Cov}\left(X_{0}, X_{k}\right)$ will converge absolutely and we will have $\lim _{n \rightarrow \infty} n^{-1} \mathbb{E}\left(S_{n}^{2}(f)\right)=\sigma^{2}$.

Since $f \in \operatorname{Mon}_{p}^{c}\left(M, P_{Y_{0}}\right)$, by definition, there exists a sequence of functions

$$
f_{L}=\sum_{k=1}^{L} a_{k, L} f_{k, L}
$$

with $f_{k, L}$ belonging to $\operatorname{Mon}_{p}\left(M, P_{Y_{0}}\right)$ and $\sum_{k=1}^{L}\left|a_{k, L}\right| \leq 1$, such that $f_{L}$ converges in $\mathbb{L}^{p}\left(P_{Y_{0}}\right)$ to $f$. Hence, $\left\|\mathbb{E}_{0}\left(X_{\ell}\right)\right\|_{p}=\lim _{L \rightarrow \infty} \| \mathbb{E}_{0}\left(f_{L}\left(Y_{\ell}\right)-\mathbb{E}\left(f_{L}\left(Y_{\ell}\right)\right)\left\|_{p} \leq \liminf _{L \rightarrow \infty} \sum_{k=1}^{L}\left|a_{k, L}\right|\right\| \mathbb{E}_{0}\left(f_{k, L}\left(Y_{\ell}\right)-\mathbb{E}\left(f_{k, L}\left(Y_{\ell}\right)\right) \|_{p}\right.\right.$. Next, by Lemma 4.3 , we have $\| \mathbb{E}_{0}\left(f_{k, L}\left(Y_{\ell}\right)-\mathbb{E}\left(f_{k, L}\left(Y_{\ell}\right)\right) \|_{p} \leq 2\left(2 \phi_{1, \mathbf{Y}}(k)\right)^{(p-1) / p} M\right.$. This shows that $\sum_{\ell \geq 0}\left\|\mathbb{E}_{0}\left(X_{\ell}\right)\right\|_{p}<\infty$ as soon as $\sum_{k \geq 1} \phi_{1, \mathbf{Y}}^{(p-1) / p}(k)<\infty$.

We shall define random variables by truncating the functions $f_{k, L}$ defined in (4.3). Let $j$ be a positive integer and

$$
g_{j}(x)=x \mathbf{1}_{|x| \leq c(j)} \text { where } c(j)=2^{j / p} j^{-2 / p} .
$$

Define then

$$
f_{L, j}=\sum_{k=1}^{L} a_{k, L} g_{j} \circ f_{k, L} .
$$

By assumption and by construction, for any integers $j, L, f_{L, j}$ has a variation bounded by $4 c(j)$. Hence, by Lemma 2.1 of $[6],\left(f_{L, j}\right)_{L}$ admits a subsequence, say $\left(f_{\varphi(L), j}\right)_{L}$ converging in $\mathbb{L}^{1}\left(P_{Y_{0}}\right)$, hence in $\mathbb{L}^{p}\left(P_{\left.Y_{0}\right)}\right)$, say to $\bar{f}_{j}$. Then, $f-\bar{f}_{j}$ is the limit in $\mathbb{L}^{p}\left(P_{Y_{0}}\right)$ of

$$
f_{\varphi(L)}-f_{\varphi(L), j}=\sum_{k=1}^{\varphi(L)} a_{k, \varphi(L)} \widetilde{g}_{j} \circ f_{k, L} \text { where } \widetilde{g}_{j}=x \mathbf{1}_{|x|>c(j)} .
$$

Define then for any integer $\ell$,

$$
\bar{X}_{j, \ell}:=\bar{f}_{j}\left(Y_{\ell}\right)-\mathbb{E}\left(\bar{f}_{j}\left(Y_{\ell}\right)\right) \text { and } \tilde{X}_{j, \ell}:=\left(f-\bar{f}_{j}\right)\left(Y_{\ell}\right)-\mathbb{E}\left(\left(f-\bar{f}_{j}\right)\left(Y_{\ell}\right)\right),
$$

and

$$
\bar{X}_{j, L, \ell}:=f_{\varphi(L), j}\left(Y_{\ell}\right)-\mathbb{E}\left(f_{\varphi(L), j}\left(Y_{\ell}\right)\right) \text { and } \tilde{X}_{j, L, \ell}:=\left(f_{\varphi(L)}-f_{\varphi(L), j}\right)\left(Y_{\ell}\right)-\mathbb{E}\left(\left(f_{\varphi(L)}-f_{\varphi(L), j}\right)\left(Y_{\ell}\right)\right) \text {. }
$$

We define also a sequence of martingale differences, $\left(\bar{d}_{j, \ell}\right)_{\ell \geq 1}$, with respect to the non-decreasing sequence of $\sigma$-algebras $\left(\mathcal{F}_{\ell}\right)_{\ell \geq 1}$, as follows:

$$
\bar{d}_{j, \ell}=\sum_{k \geq \ell} P_{\ell}\left(\bar{X}_{j, k}\right)
$$

Notice that by assumption (4.1), the series $\sum_{k \geq 0} P_{0}\left(\bar{X}_{j, k}\right)$ converges in $\mathbb{L}^{\infty}$ as shown by the following claim whose proof is given later.

Claim 1 Let $j$ be fixed. Assume that $\sum_{k \geq 1} \phi_{1, \mathbf{Y}}(k)<\infty$. Then $\sum_{k \geq 0}\left\|\mathbb{E}_{0}\left(\bar{X}_{j, k}\right)\right\|_{\infty}<\infty$, and the sequence $\left(d_{j, \ell}\right)_{\ell \geq 1}$ defined by (4.7) forms a stationary sequence of martingale differences in $\mathbb{L}^{\infty}$ with respect to the non-decreasing sequence of $\sigma$-algebras $\left(\mathcal{F}_{\ell}\right)_{\ell \geq 1}$.

We define now some non stationary sequences $\left(\bar{X}_{\ell}\right)_{\ell \geq 1}$ and $\left(\bar{d}_{\ell}\right)_{\ell \geq 1}$ as follows:

$$
\bar{d}_{1}:=\bar{d}_{1,1}, \bar{X}_{1}:=\bar{X}_{1,1},
$$

and, for every $j \geq 0$ and every $\ell \in\left\{2^{j}+1, \ldots, 2^{j+1}\right\}$,

$$
\bar{d}_{\ell}:=\bar{d}_{j, \ell}, \bar{X}_{\ell}:=\bar{X}_{j, \ell} .
$$


For every positive integer $n$, we then define

$$
\bar{M}_{n}(f):=\sum_{\ell=1}^{n} \bar{d}_{\ell} \text { and } \bar{S}_{n}(f):=\sum_{\ell=1}^{n} \bar{X}_{\ell} .
$$

With these notations, the following decomposition is valid: for any positive integer $k$,

$$
S_{k}(f)=\left(S_{k}(f)-\bar{S}_{k}(f)\right)+\left(\bar{S}_{k}(f)-\bar{M}_{k}(f)\right)+\bar{M}_{k}(f) .
$$

Therefore, the theorem will follow if we can prove that

$$
\begin{aligned}
& \sup _{1 \leq k \leq n}\left|S_{k}(f)-\bar{S}_{k}(f)\right|=o\left(n^{1 / p}(\log n)^{1-2 / p}\right) \text { almost surely, } \\
& \sup _{1 \leq k \leq n}\left|\bar{S}_{k}(f)-\bar{M}_{k}(f)\right|=o\left(n^{1 / p}(\log n)^{1-2 / p}\right) \text { almost surely, }
\end{aligned}
$$

and if, enlarging our probability space if necessary, there exists a sequence $\left(Z_{i}\right)_{i \geq 0}$ of iid Gaussian random variables with mean zero and variance $\sigma^{2}$ such that

$$
\sup _{1 \leq k \leq n}\left|\sum_{i=1}^{k}\left(\bar{d}_{i}-Z_{i}\right)\right|=o\left(n^{1 / p}(\log n)^{1-2 / p}\right) \text { almost surely. }
$$

Proof of (4.10). For any non negative integer $j$, let

$$
\widetilde{D}_{j}:=\sup _{1 \leq k \leq 2^{j}}\left|\sum_{\ell=2^{j}+1}^{k+2^{j}}\left(X_{\ell}-\bar{X}_{\ell}\right)\right|=\sup _{1 \leq k \leq 2^{j}}\left|\sum_{\ell=2^{j}+1}^{k+2^{j}} \tilde{X}_{j, \ell}\right|
$$

where $\widetilde{X}_{j, \ell}$ is defined by (4.5). Let $N \in \mathbb{N}^{*}$ and let $\left.\left.k \in\right] 1,2^{N}\right]$. We first notice that $\widetilde{D}_{j} \geq\left|\sum_{\ell=2^{j}+1}^{2^{j+1}} \widetilde{X}_{j, \ell}\right|$, so if $K$ is the integer such that $2^{K-1}<k \leq 2^{K}$, then

$$
\left|S_{k}(f)-\bar{S}_{k}(f)\right| \leq\left|X_{1}-\bar{X}_{1}\right|+\sum_{j=0}^{K-1} \widetilde{D}_{j}
$$

Consequently since $K \leq N$,

$$
\sup _{1 \leq k \leq 2^{N}}\left|S_{k}(f)-\bar{S}_{k}(f)\right| \leq\left|X_{1}-\bar{X}_{1}\right|+\sum_{j=0}^{N-1} \widetilde{D}_{j} .
$$

Therefore, (4.10) will follow if we can prove that

$$
\sup _{1 \leq k \leq 2^{j}}\left|\sum_{\ell=2^{j}+1}^{k+2^{j}} \tilde{X}_{j, \ell}\right|=o\left(j^{1-2 / p} 2^{j / p}\right) \text { a.s. }
$$

By stationarity, this will hold true as soon as

$$
\sum_{j \geq 1} \frac{\left\|\sup _{1 \leq k \leq 2^{j}}\left|\sum_{\ell=1}^{k} \tilde{X}_{j, \ell}\right|\right\|_{2}^{2}}{2^{2 j / p} j^{2-4 / p}}<\infty
$$

This is achieved by the following claim whose proof is given later.

Claim 2 Assume that

$$
\sum_{k \geq 1} k^{-1 / 2} \phi_{1, \mathbf{Y}}^{1 / 2}(k)<\infty .
$$

Then (4.14) holds. 
Proof of (4.11). For any non negative integer $j$, let

$$
\bar{D}_{j}:=\sup _{1 \leq k \leq 2^{j}}\left|\sum_{\ell=2^{j}+1}^{k+2^{j}}\left(\bar{X}_{\ell}-\bar{d}_{\ell}\right)\right|=\sup _{1 \leq k \leq 2^{j}}\left|\sum_{\ell=2^{j}+1}^{k+2^{j}}\left(\bar{X}_{j, \ell}-\bar{d}_{j, \ell}\right)\right| .
$$

Following the beginning of the proof of (4.10), we get that

$$
\sup _{1 \leq k \leq 2^{N}}\left|\bar{S}_{k}(f)-\bar{M}_{k}(f)\right| \leq\left|\bar{X}_{1}-\bar{d}_{1}\right|+\sum_{j=0}^{N-1} \bar{D}_{j} .
$$

Therefore we infer that (4.11) will hold if

$$
\sup _{1 \leq k \leq 2^{j}}\left|\sum_{\ell=2^{j}+1}^{k+2^{j}}\left(\bar{X}_{j, \ell}-\bar{d}_{j, \ell}\right)\right|=o\left(j^{1-2 / p} 2^{j / p}\right) \quad \text { a.s. }
$$

By stationarity, this will hold true as soon as

$$
\sum_{j \geq 1} \frac{\left\|\sup _{1 \leq k \leq 2^{j}}\left|\sum_{\ell=1}^{k}\left(\bar{X}_{j, \ell}-\bar{d}_{j, \ell}\right)\right|\right\|_{4}^{4}}{2^{4 j / p} j^{4(1-2 / p)}}<\infty .
$$

This is achieved by the following claim whose proof is given later.

Claim 3 Assume that

$$
\sum_{k \geq 1} k^{-1 / 8} \phi_{1, \mathbf{Y}}^{3 / 4}(k)<\infty
$$

Then (4.16) holds.

Proof OF (4.12). Let $a_{n}=n^{2 / p}(\log n)^{1-4 / p}$. The result will follow from the following claim.

Claim 4 Assume that

$$
\sum_{\ell \geq 1} a_{\ell}^{-\nu} \mathbb{E}\left(\left|\bar{d}_{\ell}\right|^{2 \nu}\right)<\infty \quad \text { for some } 1 \leq \nu \leq 2
$$

and that

$$
\sum_{i=1}^{n}\left(\mathbb{E}_{i-1}\left(\bar{d}_{i}^{2}\right)-\mathbb{E}\left(\bar{d}_{i}^{2}\right)\right)=o\left(a_{n}\right) \text { a.s. }
$$

Then (4.12) holds.

It remains to show that (4.18) and (4.19) are satisfied.

Proof of (4.18). We show it for $\nu=2$. Notice first that

$$
\sum_{\ell \geq 2} a_{\ell}^{-2} \mathbb{E}\left(\left|\bar{d}_{\ell}\right|^{4}\right)=\sum_{j \geq 0} \sum_{\ell=2^{j}+1}^{2^{j+1}} a_{\ell}^{-2} \mathbb{E}\left(\left|\bar{d}_{j, \ell}\right|^{4}\right) \ll \sum_{j \geq 0} \frac{1}{2^{4 j / p} j^{2(1-4 / p)}} \sum_{\ell=2^{j}+1}^{2^{j+1}} \mathbb{E}\left(\left|\bar{d}_{j, \ell}\right|^{4}\right)
$$

where $\bar{d}_{j, \ell}$ is defined in (4.7). By stationarity and Lemma 5.1 in [4],

$$
\left\|\bar{d}_{j, \ell}\right\|_{4} \leq \sum_{\ell \geq 0}\left\|P_{0}\left(\bar{X}_{j, \ell}\right)\right\|_{4} \ll \sum_{\ell \geq 0}(\ell+1)^{-1 / 4}\left\|\mathbb{E}_{0}\left(\bar{X}_{j, \ell}\right)\right\|_{4}
$$

Using the arguments at the beginning of the proof of Claim 1, we first observe that $\left(\bar{X}_{j, L, \ell}\right)_{L \geq 1}$ defined in (4.6) converges in $\mathbb{L}^{4}$ to $\bar{X}_{j, \ell}$. Hence

$$
\left\|\mathbb{E}_{0}\left(\bar{X}_{j, \ell}\right)\right\|_{4}=\lim _{L \rightarrow \infty}\left\|\mathbb{E}_{0}\left(\bar{X}_{j, L, \ell}\right)\right\|_{4}
$$

Next

$$
\left\|\mathbb{E}_{0}\left(\bar{X}_{j, L, \ell}\right)\right\|_{4} \leq \sum_{k=1}^{\varphi(L)}\left|a_{k, \varphi(L)}\right|\left\|\mathbb{E}_{0}\left(g_{j} \circ f_{k, \varphi(L)}\left(Y_{\ell}\right)\right)-\mathbb{E}\left(g_{j} \circ f_{k, \varphi(L)}\left(Y_{\ell}\right)\right)\right\|_{4}
$$


Applying Lemma 4.3, we then derive that

$$
\left\|\mathbb{E}_{0}\left(\bar{X}_{j, L, \ell}\right)\right\|_{4} \leq 2\left(2 \phi_{1, \mathbf{Y}}(\ell)\right)^{3 / 4} \sum_{k=1}^{\varphi(L)}\left|a_{k, \varphi(L)}\right|\left\|g_{j} \circ f_{k, \varphi(L)}\left(Y_{0}\right)\right\|_{4} .
$$

Therefore starting from (4.20), we get that

$$
\left\|\bar{d}_{j, \ell}\right\|_{4} \ll \liminf _{L \rightarrow \infty} \sum_{k=1}^{\varphi(L)}\left|a_{k, \varphi(L)}\right|\left\|g_{j} \circ f_{k, \varphi(L)}\left(Y_{0}\right)\right\|_{4} \sum_{\ell \geq 0} \ell^{-1 / 4}\left(\phi_{1, \mathbf{Y}}(k)\right)^{3 / 4} .
$$

Since $\sum_{k=1}^{\varphi(L)}\left|a_{k, \varphi(L)}\right| \leq 1$, Jensen's inequality leads to

$$
\left\|\bar{d}_{j, \ell}\right\|_{4}^{4} \ll \liminf _{L \rightarrow \infty} \sum_{k=1}^{\varphi(L)}\left|a_{k, \varphi(L)}\right|\left\|g_{j} \circ f_{k, \varphi(L)}\left(Y_{0}\right)\right\|_{4}^{4}\left(\sum_{\ell \geq 0} \ell^{-1 / 4}\left(\phi_{1, \mathbf{Y}}(\ell)\right)^{3 / 4}\right)^{4} .
$$

Hence, using condition (4.1) and Fubini, there exists a positive constant $C$ not depending on $L$ such that

$$
\begin{aligned}
& \sum_{j \geq 0} \frac{1}{2^{4 j / p} j^{2(1-4 / p)}} \sum_{\ell=2^{j}+1}^{2^{j+1}} \mathbb{E}\left(\left|\bar{d}_{j, \ell}\right|^{4}\right) \\
& \quad \ll \liminf _{L \rightarrow \infty}^{\varphi(L)} \sum_{k=1}\left|a_{k, \varphi(L)}\right| \sum_{j>0} \frac{2^{j}}{2^{4 j / p} j^{2(p-4) / p}} \mathbb{E}\left(f_{k, \varphi(L)}^{4}\left(Y_{0}\right) \mathbf{1}_{\left|f_{k, \varphi(L)}\left(Y_{0}\right)\right| \leq 2^{j / p} j-2 / p}\right) \\
& \quad \ll \liminf _{L \rightarrow \infty} \sum_{k=1}^{\varphi(L)}\left|a_{k, \varphi(L)}\right| \sum_{j>0}\left(\frac{j^{2 / p}}{2^{j / p}}\right)^{4-p} \mathbb{E}\left(f_{k, \varphi(L)}^{4}\left(Y_{0}\right) \mathbf{1}_{\left|f_{k, \varphi(L)}\left(Y_{0}\right)\right| \leq 2^{j / p} j^{-2 / p}}\right) \\
& \quad<C \liminf _{L \rightarrow \infty} \sum_{k=1}^{\varphi(L)}\left|a_{k, \varphi(L)}\right||| f_{k, \varphi(L)}\left(Y_{0}\right) \|_{p}^{p} \leq C M^{p} .
\end{aligned}
$$

This ends the proof of (4.18).

Proof of (4.19). For any non negative integer $j$, let

$$
A_{j}:=\sup _{1 \leq \ell \leq 2^{j}}\left|\sum_{i=2^{j}+1}^{2^{j}+\ell}\left(\mathbb{E}_{i-1}\left(\bar{d}_{i}^{2}\right)-\mathbb{E}\left(\bar{d}_{i}^{2}\right)\right)\right|=\sup _{1 \leq \ell \leq 2^{j}}\left|\sum_{i=2^{j}+1}^{2^{j}+\ell}\left(\mathbb{E}_{i-1}\left(\bar{d}_{j, i}^{2}\right)-\mathbb{E}\left(\bar{d}_{j, i}^{2}\right)\right)\right|
$$

where $\bar{d}_{j, i}$ is defined in (4.7). Let $N \in \mathbb{N}^{*}$ and let $\left.\left.k \in\right] 1,2^{N}\right]$. We first notice that $A_{j} \geq \mid \sum_{\ell=2^{j}+1}^{2^{j+1}}\left(\mathbb{E}_{i-1}\left(\bar{d}_{j, i}^{2}\right)-\right.$ $\left.\mathbb{E}\left(\bar{d}_{j, i}^{2}\right)\right) \mid$, so if $K$ is the integer such that $2^{K-1}<k \leq 2^{K}$, then

$$
\left|\sum_{i=1}^{k}\left(\mathbb{E}_{i-1}\left(\bar{d}_{i}^{2}\right)-\mathbb{E}\left(\bar{d}_{i}^{2}\right)\right)\right| \leq\left|\mathbb{E}_{0}\left(\bar{d}_{1}^{2}\right)-\mathbb{E}\left(\bar{d}_{1}^{2}\right)\right|+\sum_{j=0}^{N-1} A_{j}
$$

Consequently since $K \leq N$,

$$
\sup _{1 \leq k \leq 2^{N}}\left|\sum_{i=1}^{k}\left(\mathbb{E}_{i-1}\left(\bar{d}_{i}^{2}\right)-\mathbb{E}\left(\bar{d}_{i}^{2}\right)\right)\right| \leq\left|\mathbb{E}_{0}\left(\bar{d}_{1}^{2}\right)-\mathbb{E}\left(\bar{d}_{1}^{2}\right)\right|+\sum_{j=0}^{N-1} A_{j} .
$$

Therefore to prove (4.19), it is enough to show that

$$
\sup _{1 \leq k \leq 2^{j}}\left|\sum_{\ell=2^{j}+1}^{k+2^{j}}\left(\mathbb{E}_{i-1}\left(\bar{d}_{j, i}^{2}\right)-\mathbb{E}\left(\bar{d}_{j, i}^{2}\right)\right)\right| \leq 2^{2 j / p} j^{1-4 / p} \quad \text { a.s. }
$$

In particular, it suffices to prove that

$$
\sum_{j \geq 1} \frac{\left\|\sup _{1 \leq k \leq 2^{j}}\left|\sum_{i=1}^{k}\left(\mathbb{E}_{i-1}\left(\bar{d}_{j, i}^{2}\right)-\mathbb{E}\left(\bar{d}_{j, i}^{2}\right)\right)\right|\right\|_{2}^{2}}{2^{4 j / p} j^{2(1-4 / p)}}<\infty .
$$


Observe now that, by Claim 1 and condition (4.1), $\left(\bar{d}_{j, i}\right)_{i \in \mathbb{Z}}$ forms a stationary triangular sequence of martingale differences in $\mathbb{L}^{4}$. Let

$$
\bar{M}_{j, n}:=\sum_{i=1}^{n} \bar{d}_{j, i}
$$

Applying Proposition 2.3 in [22] and using the martingale property of the sequence $\left(\bar{M}_{j, n}\right)_{n \geq 1}$, we get that

$$
\left\|\sup _{1 \leq k \leq 2^{j}} \mid \sum_{i=1}^{k}\left(\mathbb{E}_{i-1}\left(\bar{d}_{j, i}^{2}\right)-\mathbb{E}\left(\bar{d}_{j, i}^{2}\right)\right)\right\|_{2}^{2} \ll 2^{j}\left\|\bar{d}_{j, 1}^{2}\right\|_{2}^{2}+2^{j}\left(\sum_{k=0}^{j-1} \frac{\left\|\mathbb{E}_{0}\left(\bar{M}_{j, 2^{k}}^{2}\right)-\mathbb{E}\left(\bar{M}_{j, 2^{k}}^{2}\right)\right\|_{2}}{2^{k / 2}}\right)^{2} .
$$

Noticing that $\left\|d_{j, 1}^{2}\right\|_{2}^{2}=\left\|d_{j, 1}\right\|_{4}^{4}$, and using the computations made in (4.22), we get that

$$
\sum_{j \geq 1} \frac{2^{j}\left\|\bar{d}_{j, 1}^{2}\right\|_{2}^{2}}{2^{4 j / p} j^{2(1-4 / p)}}<\infty
$$

Therefore to prove (4.24), it remains to prove that

$$
\sum_{j \geq 1} \frac{2^{j}}{2^{4 j / p} j^{2(1-4 / p)}}\left(\sum_{k=0}^{j-1} 2^{-k / 2}\left\|\mathbb{E}_{0}\left(\bar{M}_{j, 2^{k}}^{2}\right)-\mathbb{E}\left(\bar{M}_{j, 2^{k}}^{2}\right)\right\|_{2}\right)^{2}<\infty .
$$

Setting $\bar{S}_{j, n}=\sum_{i=1}^{n} \bar{X}_{j, i}$ and $\bar{R}_{j, n}=\bar{S}_{j, n}-\bar{M}_{j, n}$, according to the proof of Theorem 2.3 in [4], this will hold provided that

$$
\begin{gathered}
\sum_{j \geq 1} \frac{2^{j}}{2^{4 j / p} j^{2(1-4 / p)}}\left(\sum_{k=0}^{j-1} 2^{-k / 2}\left\|\mathbb{E}_{0}\left(\bar{S}_{j, 2^{k}}^{2}\right)-\mathbb{E}\left(\bar{S}_{j, 2^{k}}^{2}\right)\right\|_{2}\right)^{2}<\infty, \\
\sum_{j \geq 1} \frac{2^{j}}{2^{4 j / p} j^{2(1-4 / p)}}\left(\sum_{k=0}^{j-1} 2^{-k / 2}\left\|\bar{R}_{j, 2^{k}}\right\|_{4}^{2}\right)^{2}<\infty,
\end{gathered}
$$

and

$$
\sum_{j \geq 1} \frac{2^{j}}{2^{4 j / p} j^{2(1-4 / p)}}\left(\sum_{k=0}^{j-1} 2^{k / 2} \sum_{\ell \geq 2^{k}}\left\|P_{0}\left(\bar{X}_{j, \ell}\right)\right\|_{2}\right)^{2}<\infty .
$$

According to the proof of Claim 3,

$$
\left\|\bar{R}_{j, 2^{k}}\right\|_{4} \ll\left(\sum_{\ell \geq 1}\left(\phi_{1, Y}(\ell)\right)^{3 / 4}\right) \liminf _{L \rightarrow \infty} \sum_{i=1}^{\varphi(L)}\left|a_{i, \varphi(L)}\right|\left\|g_{j} \circ f_{i, \varphi(L)}\left(Y_{0}\right)\right\|_{4} .
$$

Therefore, since $\sum_{i=1}^{\varphi(L)}\left|a_{i, \varphi(L)}\right|<1$, using Jensen's inequality and condition (4.1),

$$
\begin{aligned}
& \sum_{j \geq 1} \frac{2^{j}}{2^{4 j / p} j^{2(1-4 / p)}}\left(\sum_{k=0}^{j-1} 2^{-k / 2}\left\|\bar{R}_{j, 2^{k}}\right\|_{4}^{2}\right)^{2} \\
& \quad \ll \liminf _{L \rightarrow \infty} \sum_{i=1}^{\varphi(L)}\left|a_{i, \varphi(L)}\right| \sum_{j \geq 1} \frac{2^{j}}{2^{4 j / p} j^{2(1-4 / p)}} \mathbb{E}\left(f_{i, \varphi(L)}^{4}\left(Y_{0}\right) \mathbf{1}_{\left|f_{i, \varphi(L)}\left(Y_{0}\right)\right| \leq 2^{j / p} j^{-2 / p}}\right)
\end{aligned}
$$

which together with an application of Fubini show (4.26). We turn to the proof of (4.27). According to Lemma 5.1 in [4],

$$
2^{k / 2} \sum_{\ell \geq 2^{k}}\left\|P_{0}\left(\bar{X}_{j, \ell}\right)\right\|_{2} \ll \sum_{\ell \geq 2^{k-1}}\left\|\mathbb{E}_{0}\left(\bar{X}_{j, \ell}\right)\right\|_{2} .
$$

Since $\left\|\mathbb{E}_{0}\left(\bar{X}_{j, \ell}\right)\right\|_{2} \leq \liminf _{L \rightarrow \infty} \sum_{i=1}^{\varphi(L)}\left|a_{i, \varphi(L)}\right|\left\|\mathbb{E}_{0}\left(g_{j} \circ f_{i, \varphi(L)}\left(Y_{\ell}\right)\right)-\mathbb{E}\left(g_{j} \circ f_{i, \varphi(L)}\left(Y_{\ell}\right)\right)\right\|_{p}$, by Lemma 4.3 and using the fact that $\sum_{i=1}^{\varphi(L)}\left|a_{i, \varphi(L)}\right| \leq 1$, we derive that

$$
\left\|\mathbb{E}_{0}\left(\bar{X}_{j, \ell}\right)\right\|_{2} \ll M\left(\phi_{1, \mathbf{Y}}(\ell)\right)^{(p-1) / p}
$$


Since $p<4$, we then infer that (4.27) will hold if $\left.\sum_{\ell>1} \phi_{1, \mathbf{Y}}(\ell)\right)^{(p-1) / p}<\infty$ which is satisfied if (4.1) is. It remains to show that (4.25) holds true. Since

$$
\left\|\mathbb{E}_{0}\left(\bar{S}_{j, 2^{k}}^{2}\right)-\mathbb{E}\left(\bar{S}_{j, 2^{k}}^{2}\right)\right\|_{2} \leq 2 \sum_{m=1}^{2^{k}} \sum_{\ell=0}^{2^{k}-m}\left\|\mathbb{E}_{0}\left(\bar{X}_{j, m} \bar{X}_{j, m+\ell}\right)-\mathbb{E}\left(\bar{X}_{j, m} \bar{X}_{j, m+\ell}\right)\right\|_{2},
$$

we infer that there exists $C>0$ such that

$$
\begin{aligned}
& \sum_{k=0}^{j-1} 2^{-k / 2}\left\|\mathbb{E}_{0}\left(\bar{S}_{j, 2^{k}}^{2}\right)-\mathbb{E}\left(\bar{S}_{j, 2^{k}}^{2}\right)\right\|_{2} \\
& \quad \leq C \sum_{m=1}^{2^{j}} \sum_{\ell=0}^{2^{j}} \frac{1}{(m+\ell)^{1 / 2}}\left\|\mathbb{E}_{0}\left(\bar{X}_{j, m} \bar{X}_{j, m+\ell}\right)-\mathbb{E}\left(\bar{X}_{j, m} \bar{X}_{j, m+\ell}\right)\right\|_{2} \\
& \quad \leq C \sum_{m=1}^{2^{j}} \sum_{\ell=0}^{\left[m^{\gamma}\right]} \frac{1}{(m+\ell)^{1 / 2}} \gamma_{j}\left(\mathcal{F}_{0}, m, \ell\right)+C \sum_{m=1}^{2^{j}} \sum_{\ell=\left[m^{\gamma}\right]}^{2^{j}} \frac{1}{(m+\ell)^{1 / 2}} \gamma_{j}\left(\mathcal{F}_{0}, m, \ell\right) .
\end{aligned}
$$

where

$$
\gamma_{j}\left(\mathcal{F}_{0}, m, \ell\right):=\left\|\mathbb{E}_{0}\left(\bar{X}_{j, m} \bar{X}_{j, m+\ell}\right)-\mathbb{E}\left(\bar{X}_{j, m} \bar{X}_{j, m+\ell}\right)\right\|_{2} \text { and } \gamma \in(0,1] .
$$

Bounding up $\gamma_{j}\left(\mathcal{F}_{0}, m, \ell\right)$ in two ways as done in the proof of Corollary 2.1 in [4], we infer that, for any $\gamma \in(0,1]$ (to be chosen later), there exists a positive constant $B$ such that

$$
\left(\sum_{k=0}^{j-1} 2^{-k / 2}\left\|\mathbb{E}_{0}\left(\bar{S}_{j, 2^{k}}^{2}\right)-\mathbb{E}\left(\bar{S}_{j, 2^{k}}^{2}\right)\right\|_{2}\right)^{2} \leq B I_{1}^{2}+B I_{2}^{2}
$$

where

$$
\begin{aligned}
& I_{1}=\sum_{m=1}^{2^{j}} \frac{m^{\gamma}}{m^{1 / 2}} \sup _{\ell \geq k \geq m}\left\|\mathbb{E}_{0}\left(\bar{X}_{j, \ell} \bar{X}_{j, k}\right)-\mathbb{E}\left(\bar{X}_{j, \ell} \bar{X}_{j, k}\right)\right\|_{2} \\
& I_{2}=\left(\sum_{k=1}^{2^{j}} \frac{k^{1 /(2 \gamma)}}{k^{1 / 4}}\left\|\mathbb{E}_{0}\left(\bar{X}_{j, k}\right)\right\|_{4}\right)^{2} .
\end{aligned}
$$

We shall proceed by using some arguments developed in [6] to get their bound (5.7). Arguing as in the proof of Claim 3, we obtain that

$$
I_{2} \leq 8 \sqrt{2}\left(\sum_{k>0} \frac{k^{1 /(2 \gamma)}}{k^{1 / 4}} \phi_{1, \mathbf{Y}}(k)^{3 / 4}\right)^{2} \liminf _{L \rightarrow \infty}\left(\sum_{\ell=1}^{\varphi(L)}\left|a_{\ell, \varphi(L)}\right| \mathbb{E}\left(f_{\ell, \varphi(L)}^{4}\left(Y_{0}\right) \mathbf{1}_{\left|f_{\ell, \varphi(L)}\left(Y_{0}\right)\right| \leq c(j)}\right)\right)^{1 / 2}
$$

We bound now $I_{1}$. According to the proof of Claim $1, \bar{X}_{j, \ell}$ is the limit in $\mathbb{L}^{4}$ of $\left(\bar{X}_{j, L, \ell}\right)_{L}$, where $\bar{X}_{j, L, \ell}$ is defined in (4.6). Therefore,

$$
\left\|\mathbb{E}_{0}\left(\bar{X}_{j, \ell} \bar{X}_{j, k}\right)-\mathbb{E}\left(\bar{X}_{j, \ell} \bar{X}_{j, k}\right)\right\|_{2}=\lim _{L \rightarrow \infty}\left\|\mathbb{E}_{0}\left(\bar{X}_{j, L, \ell} \bar{X}_{j, L, k}\right)-\mathbb{E}\left(\bar{X}_{j, L, \ell} \bar{X}_{j, L, k}\right)\right\|_{2} .
$$

Applying Lemma 4.3, for $\ell \geq k \geq m$,

$$
\begin{aligned}
\| \mathbb{E}_{0}\left(\bar{X}_{j, L, \ell} \bar{X}_{j, L, k}\right)- & \mathbb{E}\left(\bar{X}_{j, L, \ell} \bar{X}_{j, L, k}\right) \|_{2} \\
& \leq 16 \phi_{2, \mathbf{Y}}(m)^{1 / 2} \sum_{\ell=1}^{\varphi(L)}\left|a_{\ell, \varphi(L)}\right|\left\|g_{j} \circ f_{\ell, \varphi(L)}\left(Y_{0}\right)\right\|_{4} \sum_{k=1}^{\varphi(L)}\left|a_{k, \varphi(L)}\right|\left\|g_{j} \circ f_{k, \varphi(L)}\left(Y_{0}\right)\right\|_{4} .
\end{aligned}
$$

It follows that

$$
I_{1} \leq\left(16 \sum_{m=1}^{2^{j}} \frac{m^{\gamma}}{m^{1 / 2}} \phi_{2, \mathbf{Y}}(m)^{1 / 2}\right) \liminf _{L \rightarrow \infty}\left(\sum_{\ell=1}^{\varphi(L)}\left|a_{\ell, \varphi(L)}\right| \mathbb{E}\left(f_{\ell, \varphi(L)}^{4}\left(Y_{0}\right) \mathbf{1}_{\left|f_{\ell, \varphi(L)}\left(Y_{0}\right)\right| \leq c(j)}\right)\right)^{1 / 2} .
$$


Let $\gamma=1 / \sqrt{3}$. If the condition (4.1) holds, then

$$
\sum_{k>0} \frac{k^{\sqrt{3} / 2}}{k^{1 / 4}} \phi_{1, \mathbf{Y}}(k)^{3 / 4}<\infty \text { and } \sum_{m>0} \frac{m^{1 / \sqrt{3}}}{m^{1 / 2}} \phi_{2, \mathbf{Y}}(m)^{1 / 2}<\infty .
$$
that

We infer from $(4.28),(4.29)$ and (4.30) that, if (4.1) holds, there exists a positive constant $C_{4}(\phi)$ such

$$
\left(\sum_{k=0}^{j-1} 2^{-k / 2}\left\|\mathbb{E}_{0}\left(\bar{S}_{j, 2^{k}}^{2}\right)-\mathbb{E}\left(\bar{S}_{j, 2^{k}}^{2}\right)\right\|_{2}\right)^{2} \leq C_{4}(\phi) \liminf _{L \rightarrow \infty} \sum_{\ell=1}^{\varphi(L)}\left|a_{\ell, \varphi(L)}\right| \mathbb{E}\left(f_{\ell, \varphi(L)}^{4}\left(Y_{0}\right) \mathbf{1}_{\left|f_{\ell, \varphi(L)}\left(Y_{0}\right)\right| \leq c(j)}\right) .
$$

Using this last bound, Fatou's lemma together with Fubini, we then infer that (4.25) holds true. This ends the proof of (4.12).

End of the proof Theorem 4.2 for $p \in] 2,4[$. To finish the proof of Theorem 4.2 for $p \in] 2,4[$, it remains to prove our claims.

Proof of Claim 1. Notice that $\left(\bar{X}_{j, L, \ell}\right)$ converges in $\mathbb{L}^{p}$ to $\bar{X}_{j, \ell}$ and $\left\|\bar{X}_{j, L, \ell}\right\|_{\infty} \leq 2 c(j)$. Therefore, we infer that $\left\|\bar{X}_{j, \ell}\right\|_{\infty} \leq 2 c(j)$. It follows that $\bar{X}_{j, \ell}=\lim _{L \rightarrow \infty} \bar{X}_{j, L, \ell}$ in $\mathbb{L}^{q}$ for any $q \in[1, \infty[$. Next, by Lemma 4.3 and the fact that $\sum_{k=1}^{\varphi(L)}\left|a_{k, \varphi(L)}\right| \leq 1$, we get that

$$
\begin{aligned}
\left\|\mathbb{E}_{0}\left(\bar{X}_{j, L, \ell}\right)\right\|_{q} & \leq \sum_{k=1}^{\varphi(L)}\left|a_{k, \varphi(L)}\right|\left\|\mathbb{E}_{0}\left(g_{j} \circ f_{k, \varphi(L)}\left(Y_{\ell}\right)\right)-\mathbb{E}\left(g_{j} \circ f_{k, \varphi(L)}\left(Y_{\ell}\right)\right)\right\|_{\infty} \\
& \leq 4 \phi_{1, \mathbf{Y}}(\ell) \sum_{k=1}^{\varphi(L)}\left|a_{k, \varphi(L)}\right|\left\|g_{j} \circ f_{k, \varphi(L)}\left(Y_{0}\right)\right\|_{\infty} \leq 4 c(j) \phi_{1, \mathbf{Y}}(\ell) .
\end{aligned}
$$

Therefore $\left\|\mathbb{E}_{0}\left(\bar{X}_{j, \ell}\right)\right\|_{q} \leq 4 c(j) \phi_{1, \mathbf{Y}}(\ell)$. This proves the claim.

Proof of Claim 2. Let $\widetilde{S}_{j, L, k}=\sum_{\ell=1}^{k} \widetilde{X}_{j, L, \ell}$ where $\widetilde{X}_{j, L, \ell}$ is defined by (4.6). Recall that $\widetilde{X}_{j, \ell}$ is the limit in $\mathbb{L}^{p}$ of $\widetilde{X}_{j, L, \ell}$. Clearly, to prove (4.14), it suffices to prove that, there exists some positive constant $K$, such that for any positive integer $L$,

$$
\sum_{j \geq 1} \frac{\left\|\sup _{1 \leq k \leq 2^{j}}\left|\widetilde{S}_{j, L, k}\right|\right\|_{2}^{2}}{2^{2 j / p} j^{2-4 / p}}<K
$$

To prove (4.32), we use the maximal inequality of Peligrad and Utev (2006). Therefore, by stationarity,

$$
\begin{aligned}
\left\|\max _{1 \leq k \leq 2^{j}}\left|\widetilde{S}_{j, L, k}\right|\right\|_{2}^{2} & \ll 2^{j}\left\|\widetilde{X}_{j, L, 0}\right\|_{2}^{2}+2^{j}\left(\sum_{\ell=0}^{j} 2^{-\ell / 2}\left\|\mathbb{E}_{0}\left(\widetilde{S}_{j, L, 2^{\ell}}\right)\right\|_{2}\right)^{2} \\
& \ll 2^{j}\left\|\widetilde{X}_{j, L, 0}\right\|_{2}^{2}+2^{j}\left(\sum_{k=1}^{2^{j}} k^{-1 / 2}\left\|\mathbb{E}_{0}\left(\widetilde{X}_{j, L, k}\right)\right\|_{2}\right)^{2} .
\end{aligned}
$$

Notice that $\left\|\widetilde{X}_{j, L, 0}\right\|_{2}^{2} \leq 4\left(\sum_{\ell=1}^{\varphi(L)} a_{\ell, \varphi(L)}\left\|\widetilde{g}_{j} \circ f_{\ell, \varphi(L)}\left(Y_{0}\right)\right\|_{2}\right)^{2}$. Therefore, since $\sum_{\ell=1}^{L}\left|a_{\ell, L}\right| \leq 1$, by Jensen's inequality,

$$
\left\|\widetilde{X}_{j, L, 0}\right\|_{2}^{2} \leq 4 \sum_{\ell=1}^{\varphi(L)}\left|a_{\ell, \varphi(L)}\right|\left\|\widetilde{g}_{j} \circ f_{\ell, \varphi(L)}\left(Y_{0}\right)\right\|_{2}^{2}
$$

Now

$$
\left\|\mathbb{E}_{0}\left(\widetilde{X}_{j, L, k}\right)\right\|_{2} \leq \sum_{\ell=1}^{\varphi(L)}\left|a_{\ell, \varphi(L)}\right|\left\|\mathbb{E}_{0}\left(\widetilde{g}_{j} \circ f_{\ell, \varphi(L)}\left(Y_{k}\right)\right)-\mathbb{E}\left(\widetilde{g}_{j} \circ f_{\ell, \varphi(L)}\left(Y_{k}\right)\right)\right\|_{2}
$$

Applying Lemma 4.3, $\left\|\mathbb{E}_{0}\left(\widetilde{g}_{j} \circ f_{\ell, \varphi(L)}\left(Y_{k}\right)\right)-\mathbb{E}\left(\widetilde{g}_{j} \circ f_{\ell, \varphi(L)}\left(Y_{k}\right)\right)\right\|_{2} \leq 2\left(2 \phi_{1, \mathbf{Y}}(k)\right)^{1 / 2}\left\|\widetilde{g}_{j} \circ f_{\ell, \varphi(L)}\left(Y_{0}\right)\right\|_{2}$. Hence by Jensen's inequality,

$$
\left(\sum_{k=1}^{2^{j}} k^{-1 / 2}\left\|\mathbb{E}_{0}\left(\widetilde{X}_{j, L, k}\right)\right\|_{2}\right)^{2} \leq 8\left(\sum_{k \geq 1} k^{-1 / 2} \phi_{1, \mathbf{Y}}^{1 / 2}(k)\right)^{2} \sum_{\ell=1}^{\varphi(L)} \mid a_{\ell, \varphi(L)}\left\|\tilde{g}_{j} \circ f_{\ell, \varphi(L)}\left(Y_{0}\right)\right\|_{2}^{2} .
$$


Therefore, using (4.33) together with the upper bounds (4.34) and (4.35), we derive that

$$
\sum_{j \geq 1} \frac{\left\|\max _{1 \leq k \leq 2^{j}}\left|\widetilde{S}_{j, L, k}\right|\right\|_{2}^{2}}{2^{2 j / p} j^{2-4 / p}} \ll \frac{2^{j(p-2) / p}}{j^{2(p-2) / p}}\left(\sum_{k \geq 1} k^{-1 / 2} \phi_{1, \mathbf{Y}}^{1 / 2}(k)\right)^{2} \sum_{\ell=1}^{\varphi(L)}\left|a_{\ell, \varphi(L)}\right|\left\|\widetilde{g}_{j} \circ f_{\ell, \varphi(L)}\left(Y_{0}\right)\right\|_{2}^{2} .
$$

Now, via Fubini, there exists a positive constant $C$ not depending on $L$ such that

$$
\begin{aligned}
\sum_{j>0} \frac{2^{j(p-2) / p}}{j^{2(p-2) / p}}\left\|\tilde{g}_{j} \circ f_{\ell, \varphi(L)}\left(Y_{0}\right)\right\|_{2}^{2} & =\sum_{j>0} \frac{2^{j(p-2) / p}}{j^{2(p-2) / p}} \mathbb{E}\left(f_{\ell, \varphi(L)}^{2}\left(Y_{0}\right) \mathbf{1}_{\left|f_{\ell, \varphi(L)}\left(Y_{0}\right)\right|>2^{j / p} j^{-2 / p}}\right) \\
& <C\left\|f_{\ell, \varphi(L)}\left(Y_{0}\right)\right\|_{p}^{p} \leq C M^{p} .
\end{aligned}
$$

Using condition (4.15) and the fact that $\sum_{\ell=1}^{\varphi(L)}\left|a_{\ell, \varphi(L)}\right|<1$, (4.32) follows. This ends the proof of the claim.

Proof of Claim 3. For any positive integer $k$, let $\bar{S}_{j, k}=\sum_{\ell=1}^{k} \bar{X}_{j, \ell}, \bar{M}_{j, k}=\sum_{\ell=1}^{k} \bar{d}_{j, \ell}$ and

$$
\bar{R}_{j, k}=\bar{S}_{j, k}-\bar{M}_{j, k} \text {. }
$$

By stationarity, and since for any $k \geq 1, \mathbb{E}_{0}\left(\bar{M}_{j, k}\right)=0$, according to Corollary 3 in Merlevède and Peligrad [20],

$$
\begin{aligned}
\left\|\sup _{1 \leq k \leq 2^{j}}\left|\bar{R}_{j, k}\right|\right\|_{4} & \leq\left\|\bar{R}_{j, 2^{j}}\right\|_{4}+2^{j / 4} \sum_{l=0}^{j-1} 2^{-l / 4}\left\|\mathbb{E}_{0}\left(\bar{S}_{j, 2^{l}}\right)\right\|_{4} \\
& \ll\left\|\bar{R}_{j, L, 2^{j}}\right\|_{4}+2^{j / 4} \sum_{\ell=1}^{2^{j}} \ell^{-1 / 4}\left\|\mathbb{E}_{0}\left(\bar{X}_{j, \ell}\right)\right\|_{4} .
\end{aligned}
$$

Now according to item 2 of Proposition 2.1 in [4] applied with $N=2^{2 j+1}$, we get that

$$
\left\|\bar{R}_{j, 2^{j}}\right\|_{4} \ll \sum_{\ell=1}^{2^{2 j}}\left\|\mathbb{E}_{0}\left(\bar{X}_{j, \ell}\right)\right\|_{4}+2^{j / 2} \sum_{\ell \geq 2^{2 j}}\left\|P_{0}\left(\bar{X}_{j, \ell}\right)\right\|_{4}
$$

Using then Lemma 5.1 in [4], it follows that

$$
\left\|\bar{R}_{j, 2^{j}}\right\|_{4} \ll \sum_{\ell=1}^{2^{2 j+1}}\left\|\mathbb{E}_{0}\left(\bar{X}_{j, \ell}\right)\right\|_{4}+2^{j / 2} \sum_{\ell \geq 2^{2 j}} k^{-1 / 4}\left\|\mathbb{E}_{0}\left(\bar{X}_{j, \ell}\right)\right\|_{4} .
$$

So overall,

$$
\begin{aligned}
\| \sup _{1 \leq k \leq 2^{j}} & \left|\bar{R}_{j, k}\right| \|_{4} \\
& \leq \sum_{\ell=1}^{2^{2 j+1}}\left\|\mathbb{E}_{0}\left(\bar{X}_{j, \ell}\right)\right\|_{4}+2^{j / 2} \sum_{\ell \geq 2^{2 j}} k^{-1 / 4}\left\|\mathbb{E}_{0}\left(\bar{X}_{j, \ell}\right)\right\|_{4}+2^{j / 4} \sum_{\ell=1}^{2^{j}} \ell^{-1 / 4}\left\|\mathbb{E}_{0}\left(\bar{X}_{j, \ell}\right)\right\|_{4} \\
& \leq 2^{j / 4} \sum_{\ell=1}^{2^{j}} \ell^{-1 / 8}\left\|\mathbb{E}_{0}\left(\bar{X}_{j, \ell}\right)\right\|_{4}+2^{j / 2} \sum_{\ell \geq 2^{2 j}} \ell^{-1 / 4}\left\|\mathbb{E}_{0}\left(\bar{X}_{j, \ell}\right)\right\|_{4} \cdot
\end{aligned}
$$

We handle now the quantity $\left\|\mathbb{E}_{0}\left(\bar{X}_{j, \ell}\right)\right\|_{4}$. We first observe that by Lemma 4.3,

$$
\left\|\mathbb{E}_{0}\left(\bar{X}_{j, L, \ell}\right)\right\|_{4} \leq 4\left(\phi_{1, \mathbf{Y}}(\ell)\right)^{3 / 4} \sum_{k=1}^{\varphi(L)}\left|a_{k, \varphi(L)}\right|\left\|g_{j} \circ f_{k, \varphi(L)}\left(Y_{0}\right)\right\|_{4},
$$

where $X_{j, L, \ell}$ is defined in (4.6). Taking into account (4.21), (4.37) and the condition (4.17), we then infer that

$$
\left\|\sup _{1 \leq k \leq 2^{j}}\left|\bar{R}_{j, k}\right|\right\|_{4}^{4} \ll \frac{2^{j}}{2^{4 j / p} j^{4(p-2) / p}} \liminf _{L \rightarrow \infty}\left(\sum_{k=1}^{\varphi(L)}\left|a_{k, \varphi(L)}\right|\left\|g_{j} \circ f_{k, \varphi(L)}\left(Y_{0}\right)\right\|_{4}\right)^{4} .
$$


Since, $\sum_{\ell=1}^{\varphi(L)}\left|a_{\ell, \varphi(L)}\right|<1$, by Jensen's inequality

$$
\left\|\sup _{1 \leq k \leq 2^{j}}\left|\bar{R}_{j, k}\right|\right\|_{4}^{4} \ll \frac{2^{j}}{2^{4 j / p} j^{4(p-2) / p}} \liminf _{L \rightarrow \infty} \sum_{k=1}^{\varphi(L)}\left|a_{k, \varphi(L)}\right|\left\|g_{j} \circ f_{k, \varphi(L)}\left(Y_{0}\right)\right\|_{4}^{4} .
$$

Now, via Fubini, there exists a positive constant $C$ not depending on $L$ such that

$$
\begin{gathered}
\sum_{j>0} \frac{2^{j}}{2^{4 j / p} j^{4(p-2) / p}}\left\|g_{j} \circ f_{k, \varphi(L)}\left(Y_{0}\right)\right\|_{4}^{4}=\sum_{j>0} \frac{2^{j}}{2^{4 j / p} j^{4(p-2) / p}} \mathbb{E}\left(f_{\ell, \varphi(L)}^{4}\left(Y_{0}\right) \mathbf{1}_{\left|f_{\ell, \varphi(L)}\left(Y_{0}\right)\right| \leq 2^{j / p} j^{-2 / p}}\right) \\
\quad \leq \sum_{j>0}\left(\frac{2^{j / p}}{j^{2 / p}}\right)^{p-4} \mathbb{E}\left(f_{\ell, \varphi(L)}^{4}\left(Y_{0}\right) \mathbf{1}_{\left|f_{\ell, \varphi(L)}\left(Y_{0}\right)\right| \leq 2^{j / p} j^{-2 / p}}\right)<C\left\|f_{\ell, \varphi(L)}\left(Y_{0}\right)\right\|_{p}^{p} \leq C M^{p},
\end{gathered}
$$

which combined with the fact that $\sum_{\ell=1}^{\varphi(L)}\left|a_{\ell, \varphi(L)}\right|<1$ prove the claim.

Proof of Claim 4. We first prove that

$$
\sigma^{2}=\lim _{n \rightarrow \infty} n^{-1}\left\|\bar{M}_{n}(f)\right\|_{2}^{2} .
$$

Recall that (4.1) entails in particular that $\sum_{k \geq 0}\left\|P_{0}\left(X_{k}\right)\right\|_{2}<\infty$. We then define $d_{0}=\sum_{i \geq 0} P_{0}\left(f\left(Y_{0}\right) \circ \theta^{i}\right)$ and for any integer $\ell, d_{\ell}=d_{0} \circ \theta^{\ell}$. Let $M_{n}(\bar{f})=\sum_{\ell=1}^{n} d_{\ell}$. Since $\sum_{k \geq 0}\left\|P_{0}\left(X_{k}\right)\right\|_{2}<\infty$, using item 2 of Theorem 1 in [29],

$$
\left\|S_{n}(f)-M_{n}(f)\right\|_{2}=o(\sqrt{n}) .
$$

Since $\sigma^{2}=\lim _{n \rightarrow \infty} n^{-1}\left\|S_{n}(f)\right\|_{2}^{2}$, it follows from (4.39) and stationarity that $\sigma^{2}=n^{-1}\left\|M_{n}(f)\right\|_{2}^{2}=\mathbb{E}\left(d_{0}^{2}\right)$. We show now that

$$
\left\|\bar{M}_{n}(f)-M_{n}(f)\right\|_{2}=o\left(n^{1 / 2}\right) .
$$

Let $N$ be the positive integer such that $2^{N-1}<n \leq 2^{N}$. By the martingale property of $\bar{M}_{n}(f)-M_{n}(f)$ and stationarity, we have that

$$
\left\|\bar{M}_{n}(f)-M_{n}(f)\right\|_{2}^{2}=\sum_{\ell=1}^{n} \mathbb{E}\left(\left(\bar{d}_{\ell}-d_{\ell}\right)^{2}\right) \leq \mathbb{E}\left(\left(\bar{d}_{1}-d_{1}\right)^{2}\right)+\sum_{j=0}^{N-1} 2^{j} \mathbb{E}\left(\left(d_{1}-\bar{d}_{j, 1}\right)^{2}\right) .
$$

But $d_{1}-\bar{d}_{j, 1}=\sum_{\ell \geq 1} P_{1}\left(\widetilde{X}_{j, \ell}\right)$. Then, by Lemma 5.1 in [4] (see also the proof of Corollary 2 in [23]),

$$
\left\|d_{1}-\bar{d}_{j, 1}\right\|_{2} \leq \sum_{\ell \geq 0}\left\|P_{0}\left(\tilde{X}_{j, \ell}\right)\right\|_{2} \ll \sum_{\ell \geq 0}(\ell+1)^{-1 / 2}\left\|\mathbb{E}_{0}\left(\tilde{X}_{j, \ell}\right)\right\|_{2} .
$$

Let $\widetilde{X}_{j, L, \ell}$ be defined in (4.6). Applying Lemma 4.3,

$$
\left\|\mathbb{E}_{0}\left(\widetilde{X}_{j, L, \ell}\right)\right\|_{2} \leq 2 \sqrt{2}\left(\phi_{1, \mathbf{Y}}(\ell)\right)^{1 / 2} \sum_{k=1}^{\varphi(L)}\left|a_{k, \varphi(L)}\right|\left\|\widetilde{g}_{j} \circ f_{k, \varphi(L)}\left(Y_{0}\right)\right\|_{2} .
$$

Since $\sum_{k=1}^{\varphi(L)}\left|a_{k, \varphi(L)}\right| \leq 1$ and $f_{k, \varphi(L)}$ belongs to $\operatorname{Mon}_{p}\left(M, P_{Y_{0}}\right)$, it follows that

$$
\left\|\mathbb{E}_{0}\left(\widetilde{X}_{j, L, \ell}\right)\right\|_{2} \leq 2 \sqrt{2} M^{p / 2}(c(j))^{1-p / 2}\left(\phi_{1, \mathbf{Y}}(k)\right)^{1 / 2} .
$$

Since $\left\|\mathbb{E}_{0}\left(\widetilde{X}_{j, \ell}\right)\right\|_{2}=\lim _{L \rightarrow \infty}\left\|\mathbb{E}_{0}\left(\widetilde{X}_{j, L, \ell}\right)\right\|_{2}$ and, by condition (4.1), $\sum_{k \geq 1} k^{-1 / 2} \phi_{1, \mathbf{Y}}^{1 / 2}(k)<\infty$, we get overall that

$$
\left\|\bar{M}_{n}(f)-M_{n}(f)\right\|_{2}^{2} \ll \sum_{j=0}^{N-1} 2^{j}(c(j))^{2-p} \ll 2^{2 N / p} N^{2(1-2 / p)},
$$

proving (4.40). Combining the fact that $\sigma^{2}=n^{-1}\left\|M_{n}(f)\right\|_{2}^{2}$ with (4.40), it follows that (4.38) holds.

Then, according to Theorem 2.1 in [26], we see that, enlarging our probability space if necessary, one may find a sequence $\left(\bar{Z}_{\ell}\right)_{\ell \geq 1}$ of independent Gaussian random variables with zero mean and variance $\mathbb{E}\left(\bar{Z}_{\ell}\right)^{2}=\mathbb{E}\left(\bar{d}_{\ell}\right)^{2}=\left(\bar{\sigma}_{\ell}\right)^{2}$ such that

$$
\sup _{1 \leq k \leq n}\left|\bar{M}_{k}-\sum_{\ell=1}^{k} \bar{Z}_{\ell}\right|=o\left(a_{n}^{1 / 2}(\log n)^{1 / 2}\right) \text { almost surely, as } n \rightarrow \infty .
$$


Let $\left(\delta_{k}\right)_{k \geq 1}$ be a sequence of iid Gaussian random variables with mean zero and variance $\sigma^{2}$, independent of the sequence $\left(\bar{Z}_{\ell}\right)_{\ell \geq 1}$. We now construct a sequence $\left(Z_{\ell}\right)_{\ell \geq 1}$ as follows. If $\bar{\sigma}_{\ell}=0$, then $Z_{\ell}=\delta_{\ell}$, else $Z_{\ell}=\left(\sigma / \bar{\sigma}_{\ell}\right) \bar{Z}_{\ell}$. By construction, the $Z_{\ell}$ 's are iid Gaussian random variables with mean zero and variance $\sigma^{2}$. Let $G_{\ell}=Z_{\ell}-\bar{Z}_{\ell}$ and note that $\left(G_{\ell}\right)_{\ell \geq 1}$ is a sequence of independent Gaussian random variables with mean zero and variances $\operatorname{Var}\left(G_{\ell}\right)=\left(\sigma-\bar{\sigma}_{\ell}\right)^{2}$. Assume that we can prove that

$$
\sum_{n \geq 3} \frac{\mathbb{E}\left(G_{n}^{2}\right)}{a_{n} \log n}<\infty
$$

Then by the Kolmogorov theorem (or Lemma 6.2), it will follow that the series $\sum_{n \geq 3} \frac{G_{n}}{\left(a_{n} \log n\right)^{1 / 2}}$ converges $\mathbb{P}$-a.s. Hence, Kronecker lemma will imply that $\sum_{\ell=1}^{n} G_{\ell}=o\left(\left(a_{n} \log n\right)^{1 / 2}\right) \mathbb{P}$-a.s. Therefore starting from (4.42), we will conclude that if (4.18) and (4.19) hold then (4.12) does. Let us prove (4.43). With this aim, we first notice that

$$
\mathbb{E}\left(G_{n}^{2}\right)=\left(\left\|d_{n}\right\|_{2}-\left\|\bar{d}_{n}\right\|_{2}\right)^{2} \leq\left\|d_{n}-\bar{d}_{n}\right\|_{2}^{2}
$$

Next

$$
\sum_{n \geq 3} \frac{\mathbb{E}\left(G_{n}^{2}\right)}{a_{n} \log n} \leq \sum_{j \geq 1} \frac{1}{2^{2 j / p} j^{2-4 / p}} \sum_{\ell=2^{j}+1}^{2^{j+1}} \mathbb{E}\left(\left(d_{\ell}-\bar{d}_{j, \ell}\right)^{2}\right)=\sum_{j \geq 1} \frac{2^{j}}{2^{2 j / p} j^{2-4 / p}} \mathbb{E}\left(\left(d_{1}-\bar{d}_{j, 1}\right)^{2}\right) .
$$

Using the computations as done to prove (4.40), we infer that under condition (4.1),

$$
\sum_{n \geq 3} \frac{\mathbb{E}\left(G_{n}^{2}\right)}{a_{n} \log n} \ll \liminf _{L \rightarrow \infty} \sum_{j \geq 1} \frac{2^{j}}{2^{2 j / p} j^{2-4 / p}}\left(\sum_{k=1}^{\varphi(L)}\left|a_{k, \varphi(L)}\right|\left\|\widetilde{g}_{j} \circ f_{k, \varphi(L)}\left(Y_{0}\right)\right\|_{2}\right)^{2} .
$$

Since $\sum_{k=1}^{\varphi(L)}\left|a_{k, \varphi(L)}\right| \leq 1$, Jensen's inequality leads to

$$
\sum_{n \geq 3} \frac{\mathbb{E}\left(G_{n}^{2}\right)}{a_{n} \log n} \ll \liminf _{L \rightarrow \infty} \sum_{j \geq 1} \frac{2^{j}}{2^{2 j / p} j^{2-4 / p}} \sum_{k=1}^{\varphi(L)}\left|a_{k, \varphi(L)}\right|\left\|\widetilde{g}_{j} \circ f_{k, \varphi(L)}\left(Y_{0}\right)\right\|_{2}^{2} .
$$

Hence, by Fubini theorem, there exists a positive constant $C$ not depending on $L$ such that

$$
\begin{aligned}
\sum_{n \geq 3} \frac{\mathbb{E}\left(G_{n}^{2}\right)}{a_{n} \log n} & \ll \liminf _{L \rightarrow \infty} \sum_{j \geq 1} \frac{2^{j}}{2^{2 j / p} j^{2-4 / p}} \sum_{k=1}^{\varphi(L)}\left|a_{k, \varphi(L)}\right|\left\|\widetilde{g}_{j} \circ f_{k, \varphi(L)}\left(Y_{0}\right)\right\|_{2}^{2} \\
& \ll \liminf _{L \rightarrow \infty} \sum_{k=1}^{\varphi(L)}\left|a_{k, \varphi(L)}\right| \sum_{j \geq 1}\left(\frac{2^{j / p}}{j^{2 / p}}\right)^{p-2} \mathbb{E}\left(f_{k, \varphi(L)}^{2}\left(Y_{0}\right) \mathbf{1}_{\left.\left|f_{k, \varphi(L)}\left(Y_{0}\right)\right|>2^{j / p} j^{-2 / p}\right)}\right) \\
& <C \liminf _{L \rightarrow \infty} \sum_{k=1}^{\varphi(L)}\left|a_{k, \varphi(L)}\right|\left\|f_{k, \varphi(L)}\left(Y_{0}\right)\right\|_{p}^{p} \leq C M^{p} .
\end{aligned}
$$

This ends the proof of (4.43) and of Claim 4.

\subsection{Proof of Theorem 4.2 for $p=4$}

In this case, no truncation is needed. The beginning of the proof of Theorem 4.2 for $2<p<4$ also works for $p=4$. In particular, if $f \in \operatorname{Mon}_{4}^{c}\left(M, P_{Y_{0}}\right)$, condition (4.1) implies that $\sum_{\ell>0}\left\|\mathbb{E}_{0}\left(X_{\ell}\right)\right\|_{4}<\infty$. Therefore the series $\sigma^{2}=\sum_{k \in \mathbb{Z}} \operatorname{Cov}\left(X_{0}, X_{k}\right)$ converges absolutely and $\lim _{n \rightarrow \infty} n^{-1} \mathbb{E}\left(S_{n}^{2}(f)\right)=\sigma^{2}$. In addition the series $\sum_{k \geq 0} P_{0}\left(X_{k}\right)$ converges in $\mathbb{L}^{4}$ and the sequence $\left(d_{\ell}\right)_{\ell \in \mathbb{Z}}$ defined by:

$$
d_{\ell}=\sum_{k \geq \ell} P_{\ell}\left(X_{k}\right)
$$

forms a stationary sequence of martingale differences in $\mathbb{L}^{4}$ with respect to the non-decreasing sequence of $\sigma$-algebras $\left(\mathcal{F}_{\ell}\right)_{\ell \in \mathbb{Z}}$. Hence, setting for every positive integer $n$,

$$
M_{n}(f):=\sum_{\ell=1}^{n} d_{\ell} \text { and } S_{n}(f):=\sum_{\ell=1}^{n} X_{\ell},
$$


the conclusion of Theorem 4.2 when $p=4$ will follow if we can prove that

$$
\sup _{1 \leq k \leq n}\left|S_{n}(f)-M_{n}(f)\right|=O\left(n^{1 / 4}(\log n)^{1 / 2}(\log \log n)^{1 / 4}\right) \text { almost surely, }
$$

and if, enlarging our probability space if necessary, there exists a sequence $\left(Z_{i}\right)_{i \geq 0}$ of iid Gaussian random variables with mean zero and variance $\sigma^{2}$ such that

$$
\sup _{1 \leq k \leq n}\left|\sum_{i=1}^{k}\left(d_{i}-Z_{i}\right)\right|=O\left(n^{1 / 4}(\log n)^{1 / 2}(\log \log n)^{1 / 4}\right) \text { almost surely. }
$$

To prove (4.45), it suffices to notice that since $\sum_{\ell>0}\left\|\mathbb{E}_{0}\left(X_{\ell}\right)\right\|_{4}<\infty$, we have the coboundary decomposition $S_{n}(f)=M_{n}(f)+r_{0}-r_{0} \circ \theta^{n}$ with $\left\|r_{0}\right\|_{4}<\infty$. So (4.45) follows directly from the fact that $\left(r_{0}-r_{0} \circ \theta^{n}\right) / n^{1 / 4} \rightarrow 0 \mathbb{P}$-a.s. To prove (4.46), we shall use Remark 2.9. Therefore we need to show that

$$
\sum_{k=1}^{n}\left(\mathbb{E}\left(d_{k}^{2} \mid \mathcal{F}_{k-1}\right)-\mathbb{E}\left(d_{k}^{2}\right)\right)=O\left(n^{1 / 2}(\log \log n)^{1 / 2}\right) \quad \mathbb{P} \text {-a.s. }
$$

This condition follows directly from Theorem 12 of [19] together with the fact that $\left(d_{k}\right)_{k \in \mathbb{N}}$ is a martingale differences sequence provided that

$$
\sum_{n \geq 2} \frac{(\log n)^{3}}{n^{2}}\left\|\mathbb{E}_{0}\left(M_{n}^{2}\right)-\mathbb{E}\left(M_{n}^{2}\right)\right\|_{2}^{2}<\infty
$$

According to the proof of Theorem 2.3 and Corollary 2.1 in [4], this will hold true provided that there exists $\gamma \in] 0,1]$ such that

$$
\sum_{n>0}(\log n)^{3} n^{\frac{1}{\gamma}+\frac{1}{2}}\left\|\mathbb{E}_{0}\left(X_{n}\right)\right\|_{4}^{2}<\infty
$$

and

$$
\sum_{n>0}(\log n)^{3} n^{2 \gamma} \sup _{i \geq j \geq n}\left\|\mathbb{E}_{0}\left(X_{i} X_{j}\right)-\mathbb{E}\left(X_{i} X_{j}\right)\right\|_{2}^{2}<\infty .
$$

Notice that $\left\|\mathbb{E}_{0}\left(X_{n}\right)\right\|_{4}=\lim _{L \rightarrow \infty}\left\|\mathbb{E}_{0}\left(f_{L}\left(Y_{n}\right)-\mathbb{E}\left(f_{L}\left(Y_{n}\right)\right)\right)\right\|_{4} \leq \liminf _{L \rightarrow \infty} \sum_{k=1}^{L}\left|a_{k, L}\right| \| \mathbb{E}_{0}\left(f_{k, L}\left(Y_{n}\right)-\right.$ $\mathbb{E}\left(f_{k, L}\left(Y_{n}\right)\right) \|_{4}$. Next, by Lemma 4.3, $\| \mathbb{E}_{0}\left(f_{k, L}\left(Y_{n}\right)-\mathbb{E}\left(f_{k, L}\left(Y_{n}\right)\right) \|_{4} \leq 2 M\left(2 \phi_{1, Y}(n)\right)^{3 / 4}\right.$. On the other hand,

$$
\left\|\mathbb{E}_{0}\left(X_{i} X_{j}\right)-\mathbb{E}\left(X_{i} X_{j}\right)\right\|_{2} \leq \liminf _{L \rightarrow \infty} \sum_{k=1}^{L} \sum_{\ell=1}^{L}\left|a _ { k , L } \| a _ { \ell , L } | \| \mathbb { E } _ { 0 } \left(f_{k, L}\left(Y_{i}\right) f_{\ell, L}\left(Y_{j}\right)-\mathbb{E}\left(f_{k, L}\left(Y_{i}\right) f_{\ell, L}\left(Y_{j}\right) \|_{2}\right.\right.\right.
$$

and by Lemma 4.3

$$
\sup _{i \geq j \geq n} \| \mathbb{E}_{0}\left(f_{k, L}\left(Y_{i}\right) f_{\ell, L}\left(Y_{j}\right)-\mathbb{E}\left(f_{k, L}\left(Y_{i}\right) f_{\ell, L}\left(Y_{j}\right) \|_{2} \leq 16 M^{2}\left(\phi_{2, \mathbf{Y}}(n)\right)^{1 / 2} .\right.\right.
$$

Hence condition (4.1) (for $p=4$ ) implies that (4.48) and (4.49) hold for $\gamma=1 / \sqrt{3}$. The proof of Theorem 4.2 is therefore complete.

\section{Proof of the results of Section 3}

\subsection{Proof of Theorem 3.4 on uniformly expanding maps}

Item 1 follows directly from Theorem 4.2. Indeed since $T$ is uniformly expanding, it follows from Section 6.3 in [8] that

$$
\phi_{2, \mathbf{Y}}(n)=O\left(\rho^{n}\right) \text { for some } \rho \in(0,1) .
$$

To prove Item 2 we proceed as follows. We start by the case $p \in] 2,4\left[\right.$. Since $f \in \operatorname{Mon}_{p}^{c}(M, \nu)$, we consider the function $f_{j}$ and the random variables $\bar{X}_{j, k}$ and $\widetilde{X}_{j, k}$ defined in the beginning of the proof of Theorem 4.2. In addition we set

$$
\mathcal{F}_{k}=\sigma\left(Y_{i}, i \leq k\right) \text { and } \mathcal{G}_{k}=\sigma\left(T^{i}, i \geq k\right) .
$$


As in the proof of Theorem 4.2, we define a sequence of martingale differences, $\left(\bar{d}_{j, \ell}\right)_{\ell \geq 1}$, with respect to the non-decreasing sequence of $\sigma$-algebras $\left(\mathcal{F}_{\ell}\right)_{\ell \geq 1}$, as follows:

$$
\bar{d}_{j, \ell}=\sum_{k \geq \ell}\left(\mathbb{E}\left(\bar{X}_{j, k} \mid \mathcal{F}_{\ell}\right)-\mathbb{E}\left(\bar{X}_{j, k} \mid \mathcal{F}_{\ell-1}\right)\right):=\sum_{k \geq \ell} P_{\ell}\left(\bar{X}_{j, k}\right)
$$

and we recall that by Claim 1 and by (5.1), the series $\sum_{k \geq 0} P_{0}\left(\bar{X}_{j, k}\right)$ converges in $\mathbb{L}^{\infty}$. Notice now that by the Markovian property of $\left(Y_{i}\right)_{i \geq 0}$, we have that

$$
\bar{d}_{j, \ell}=\sum_{k \geq \ell}\left(\mathbb{E}\left(\bar{X}_{j, k} \mid Y_{\ell}\right)-\mathbb{E}\left(\bar{X}_{j, k} \mid Y_{\ell-1}\right)\right):=m\left(Y_{\ell}, Y_{\ell-1}\right),
$$

where $m(\cdot, \cdot)$ is a measurable function from $\mathbb{R}^{2}$ to $\mathbb{R}$. Define now

$$
d_{j, \ell}^{*}=m\left(T^{\ell-1}, T^{\ell}\right)
$$

Notice then that $d_{j, \ell}^{*}$ is $\mathcal{G}_{\ell-1}$-measurable. Moreover, since on the probability space $([0,1], \nu)$, the random vector $\left(T, T^{2}, \ldots, T^{n}\right)$ is distributed as $\left(Y_{n}, Y_{n-1}, \ldots, Y_{1}\right)$, we have that

$$
\left\|\mathbb{E}\left(d_{j, \ell}^{*} \mid \mathcal{G}_{\ell}\right)\right\|_{1, \nu}=\left\|\mathbb{E}\left(d_{j, \ell}^{*} \mid T^{\ell}\right)\right\|_{1, \nu}=\left\|\mathbb{E}\left(\bar{d}_{j, \ell} \mid Y_{\ell-1}\right) \mid\right\|_{1}=0,
$$

it follows that $\mathbb{E}\left(d_{j, \ell}^{*} \mid \mathcal{G}_{\ell}\right)=0 \nu$-a.s.

We define now some non stationary sequences $\left(X_{\ell}^{*}\right)_{\ell \geq 1}$ and $\left(d_{\ell}^{*}\right)_{\ell \geq 1}$ as follows:

$$
d_{1}^{*}:=d_{1,1}^{*}, X_{1}^{*}:=\bar{f}_{1} \circ T-\nu\left(\bar{f}_{1} \circ T\right),
$$

and, for every $j \geq 0$ and every $\ell \in\left\{2^{j}+1, \ldots, 2^{j+1}\right\}$,

$$
d_{\ell}^{*}:=d_{j, \ell}^{*}, X_{\ell}^{*}:=\bar{f}_{j} \circ T^{\ell}-\nu\left(\bar{f}_{j} \circ T^{\ell}\right) .
$$

For every positive integer $n$, we then define

$$
M_{n}^{*}(f):=\sum_{\ell=1}^{n} d_{\ell}^{*} \text { and } S_{n}^{*}(f):=\sum_{\ell=1}^{n} X_{\ell}^{*} .
$$

Therefore, Item 2 of Theorem 3.4 will follow if we can prove that

$$
\begin{aligned}
\sup _{1 \leq k \leq n} & \left|\sum_{i=1}^{k}\left(f \circ T^{i}-\nu(f)\right)-\bar{S}_{k}^{*}(f)\right|=o\left(n^{1 / p}(\log n)^{1-2 / p}\right) \nu \text {-a.s. } \\
& \sup _{1 \leq k \leq n}\left|\bar{S}_{k}^{*}(f)-\bar{M}_{k}^{*}(f)\right|=o\left(n^{1 / p}(\log n)^{1-2 / p}\right) \nu \text {-a.s. }
\end{aligned}
$$

and if, enlarging our probability space if necessary, there exists a sequence $\left(Z_{i}^{*}\right)_{i \geq 0}$ of iid Gaussian random variables with mean zero and variance $\sigma^{2}$ such that

$$
\sup _{1 \leq k \leq n}\left|\sum_{i=1}^{k}\left(d_{i}^{*}-Z_{i}^{*}\right)\right|=o\left(n^{1 / p}(\log n)^{1-2 / p}\right) \nu \text {-a.s. }
$$

According to the proof of Theorem 4.2, (5.7) will hold if

$$
\sum_{j>0} 2^{-2 j / p} j^{-2+4 / p}\left\|\max _{1 \leq k \leq 2^{j}}\left|\sum_{\ell=1}^{k}\left(\left(f-\bar{f}_{j}\right) \circ T^{\ell}-\nu\left(f-\bar{f}_{j}\right)\right)\right|\right\|_{2, \nu}^{2}<\infty .
$$

But, since on the probability space $([0,1], \nu)$, the random vector $\left(T, T^{2}, \ldots, T^{n}\right)$ is distributed as $\left(Y_{n}, Y_{n-1}, \ldots, Y_{1}\right)$, according to the inequality (4.1) in [5], we have

$$
\left.\nu\left(\max _{1 \leq k \leq n} \mid \sum_{\ell=1}^{k}\left(\left(f-\bar{f}_{j}\right) \circ T^{\ell}-\nu\left(f-\bar{f}_{j}\right)\right)\right) \mid>x\right) \leq \mathbb{P}\left(2 \max _{1 \leq k \leq n}\left|\sum_{\ell=1}^{k} \widetilde{X}_{j, \ell}\right|>x\right) .
$$


Therefore (5.10) follows from Claim 2.

We turn now to the proof of (5.8). According to the proof of Theorem 4.2, (5.8) will hold if

$$
\sum_{j>0} 2^{-4 j / p} j^{-4(1-2 / p)}\left\|\max _{1 \leq k \leq 2^{j}}\left|\sum_{\ell=1}^{k}\left(\bar{f}_{j} \circ T^{\ell}-\nu\left(\bar{f}_{j}\right)-d_{j, \ell}^{*}\right)\right|\right\|_{4, \nu}^{4}<\infty .
$$

But, as before, since on the probability space $([0,1], \nu)$, the random vector $\left(T, T^{2}, \ldots, T^{n}\right)$ is distributed as $\left(Y_{n}, Y_{n-1}, \ldots, Y_{1}\right)$,

$$
\left.\nu\left(\max _{1 \leq k \leq 2^{j}} \mid \sum_{\ell=1}^{k}\left(\bar{f}_{j} \circ T^{\ell}-\nu\left(\bar{f}_{j}\right)-d_{j, \ell}^{*}\right)\right) \mid>x\right) \leq \mathbb{P}\left(2 \max _{1 \leq k \leq n}\left|\sum_{\ell=1}^{k}\left(\bar{X}_{j, \ell}-\bar{d}_{j, \ell}\right)\right|>x\right) .
$$

Therefore (5.11) follows from Claim 3.

To prove (5.9), we shall proceed as for the proof of (4.12) with the difference that Theorem 2.3 is used instead of Theorem 2.1 in [26]. So we have to prove that

$$
\sum_{i=1}^{n}\left(\mathbb{E}\left(\left(d_{i}^{*}\right)^{2} \mid T^{i}\right)-\mathbb{E}\left(\left(d_{i}^{*}\right)^{2}\right)\right)=o\left(a_{n}\right) \text { a.s. }
$$

where $a_{n}=n^{2 / p}(\log n)^{1-4 / p}$. But following the proof of (4.19), this will follow if we can prove that

$$
\sum_{j>0} 2^{-4 j / p} j^{-4(1-2 / p)}\left\|\max _{1 \leq k \leq 2^{j}}\left|\sum_{i=1}^{k}\left(\mathbb{E}\left(\left(d_{j, i}^{*}\right)^{2} \mid T^{i}\right)-\mathbb{E}\left(\left(d_{j, i}^{*}\right)^{2}\right)\right)\right|\right\|_{2, \nu}^{2}<\infty .
$$

But using again the fact that, on the probability space $([0,1], \nu)$, the random vector $\left(T, T^{2}, \ldots, T^{n}\right)$ is distributed as $\left(Y_{n}, Y_{n-1}, \ldots, Y_{1}\right)$, we have that

$$
\nu\left(\max _{1 \leq k \leq 2^{j}}\left|\sum_{i=1}^{k}\left(\mathbb{E}\left(\left(d_{j, i}^{*}\right)^{2} \mid T^{i}\right)-\mathbb{E}\left(\left(d_{j, i}^{*}\right)^{2}\right)\right)\right|>x\right) \leq \mathbb{P}\left(2 \max _{1 \leq k \leq 2^{j}}\left|\sum_{i=1}^{k}\left(\mathbb{E}_{i-1}\left(\bar{d}_{j, i}^{2}\right)-\mathbb{E}\left(\bar{d}_{j, i}^{2}\right)\right)\right|>x\right)
$$

Therefore (5.12) follows by the fact that (4.24) holds true. This ends the proof of Item 2 of Theorem 3.4 when $p \in] 2,4[$.

We turn now to the proof of Item 2 when $p=4$. Let $X_{i}=f\left(Y_{i}\right)-\nu(f)$. According to the beginning of the proof of Theorem 4.2, (5.1) implies that $\sum_{k>1}\left\|\mathbb{E}\left(X_{k} \mid \mathcal{F}_{0}\right)\right\|_{4}<\infty$. We define a sequence $\left(d_{\ell}\right)_{\ell \geq 1}$ of stationary martingale differences with respect to the non-decreasing sequence of $\sigma$-algebras $\left(\mathcal{F}_{\ell}\right)_{\ell \geq 1}$ and that are in $\mathbb{L}^{4}$ as follows:

$$
d_{\ell}=\sum_{k \geq \ell} P_{\ell}\left(X_{k}\right)
$$

By the Markovian property of $\left(Y_{i}\right)_{i \geq 0}$,

$$
d_{\ell}=\sum_{k \geq \ell}\left(\mathbb{E}\left(X_{k} \mid Y_{\ell}\right)-\mathbb{E}\left(X_{k} \mid Y_{\ell-1}\right)\right):=m\left(Y_{\ell}, Y_{\ell-1}\right),
$$

where $m(\cdot, \cdot)$ is a measurable function from $\mathbb{R}^{2}$ to $\mathbb{R}$. We define now

$$
d_{\ell}^{*}=m\left(T^{\ell-1}, T^{\ell}\right) .
$$

As before, notice that $d_{\ell}^{*}$ is $\mathcal{G}_{\ell-1}$-measurable and satisfies $\mathbb{E}\left(d_{j, \ell}^{*} \mid \mathcal{G}_{\ell}\right)=0 \nu$-a.s. According to Corollary 2.7 , the result will then follow if we can prove that

$$
\sum_{k=1}^{n}\left(\mathbb{E}\left(\left(d_{k}^{*}\right)^{2} \mid \mathcal{G}_{k}\right)-\mathbb{E}\left(\left(d_{k}^{*}\right)^{2}\right)\right)=O\left(n^{1 / 2}(\log \log n)^{1 / 2}\right) \quad \nu \text {-a.s. }
$$

But $\mathbb{E}\left(\left(d_{k}^{*}\right)^{2} \mid \mathcal{G}_{k}\right)=\mathbb{E}\left(\left(d_{k}^{*}\right)^{2} \mid T^{k}\right):=h\left(T^{k}\right) \nu$-a.s. where $h(\cdot)$ is a measurable function such that $\nu\left(h^{2}\right)<\infty$. Let $\widetilde{h}=h-\nu(h)$. Assume that we can prove that

$$
\sum_{n \geq 1}(\log n)^{3} \frac{\left\|\widetilde{h}+K \widetilde{h}+\cdots+K^{n-1} \widetilde{h}\right\|_{2, \nu}^{2}}{n^{2}}<\infty .
$$


This condition implies in particular that $\sum_{n \geq 1} \frac{\left\|\widetilde{h}+K \widetilde{h}+\cdots+K^{n-1} \widetilde{h}\right\|_{2, \nu}}{n^{3 / 2}}<\infty$. By Lemma 2 of [19] (and its proof $)$, it follows that $\varphi\left(Y_{0}, Y_{1}\right):=\lim _{n} \frac{1}{n} \sum_{j=1}^{n} \sum_{k=0}^{j-1}\left(K^{k} \widetilde{h}\left(Y_{1}\right)-K^{k} \widetilde{h}\left(Y_{0}\right)\right)$ exists in $\mathbb{L}^{2}$ and $M_{n}(\varphi):=$ $\sum_{k=1}^{n} \varphi\left(Y_{k-1}, Y_{k}\right)$ is a martingale with stationary increments such that

$$
\left\|\widetilde{h}\left(Y_{1}\right)+\cdots+\widetilde{h}\left(Y_{n}\right)-M_{n}(\varphi)\right\|_{2} \ll \sqrt{n} \sum_{k \geq n} \frac{\left\|\widetilde{h}+K \widetilde{h}+\cdots+K^{k-1} \widetilde{h}\right\|_{2, \nu}}{k^{3 / 2}} .
$$

Since the random vectors $\left(I d, T, T^{2}, \ldots, T^{n-1}\right)$ and $\left(Y_{n}, Y_{n-1}, \ldots, Y_{1}\right)$ have same distribution, we can write $M_{n}^{*}(\varphi):=\sum_{k=1}^{n} \varphi\left(T^{k}, T^{k-1}\right)=\sum_{k=0}^{n-1} \varphi\left(T^{n-k}, T^{n-k-1}\right)$. Then $\left(M_{n}^{*}\right)$ is a sum associated to a stationary sequence of reverse martingale differences and

$$
\left\|\widetilde{h}+\cdots+\widetilde{h} \circ T^{n-1}-M_{n}^{*}(\varphi)\right\|_{2, \nu} \ll \sqrt{n} \sum_{k \geq n} \frac{\left\|\widetilde{h}+K \widetilde{h}+\cdots+K^{k-1} \widetilde{h}\right\|_{2, \nu}}{k^{3 / 2}} .
$$

Therefore, by Corollary 4.2 of [3] with $b(n)=\log n$, if

$$
\sum_{n} \frac{\log n}{n^{2}}\left\|\widetilde{h}+\cdots+\widetilde{h} \circ T^{n-1}-M_{n}^{*}(\varphi)\right\|_{2, \nu}^{2}<\infty
$$

then

$$
\frac{\widetilde{h}+\cdots+\widetilde{h} \circ T^{n-1}-M_{n}^{*}(\varphi)}{\sqrt{n \log \log n}} \rightarrow 0 \quad \nu \text {-a.s. }
$$

Using (5.15), it is easy to see that (5.16) holds as soon as (5.14) is satisfied. Using then Corollary 2.5 to observe that $M_{n}^{*}(\varphi)=O(\sqrt{n \log \log n}) \nu$-a.s., we conclude that $(5.13)$ (and then Item 2 when $p=4$ ) holds as soon as (5.14) does. Notice now that (5.14) can be rewritten as

$$
\sum_{n \geq 1}(\log n)^{3} \frac{\left\|\sum_{k=1}^{n}\left(\mathbb{E}\left(d_{k}^{2} \mid \mathcal{F}_{0}\right)-\mathbb{E}\left(d_{k}^{2}\right)\right)\right\|_{2}^{2}}{n^{2}}<\infty
$$

that is exactly condition (4.47).

\subsection{Proof of Theorem 3.5}

The proof follows directly by analyzing the proof of Theorem 3.4 when $p=4$. Indeed, the proof reveals that if the conditions (4.48) and (4.49) hold for $X_{i}=f\left(Y_{i}\right)-\nu(f)$, then the strong approximation principle holds for both $\sum_{i=1}^{n}\left(f\left(Y_{i}\right)-\nu(f)\right)$ and $\sum_{i=1}^{n}\left(f \circ T^{i}-\nu(f)\right)$ with rate $O\left(n^{1 / 4}(\log n)^{1 / 2}(\log n \log n)^{1 / 4}\right)$. The condition (3.2) (resp. (3.3)) is exactly the condition (4.48) (resp. (4.49)) rewritten with the help of the transition operator $K$.

\subsection{Proof of Theorem 3.7 on uniformly expanding maps}

It suffices to verify the assumptions of Theorem 3.5. Using the first part of Condition (3.4) and the fact that $|f(x)-f(y)| \leq c(|x-y|)$ where $c$ is a concave and non-decreasing function, it follows from Lemma 17 in [7] that $\left\|K^{n}(f)-\nu(f)\right\|_{\infty, \nu} \leq c\left(C \rho^{n}\right)$. Therefore, (3.2) is satisfied with $\gamma=1 / \sqrt{3}$ as soon as (3.5) is. To verify now the condition (3.3) of Theorem 3.5, we shall use similar arguments as those developed in the proof of Corollary 3.12 in [4]. From Section 7 in [9], we know that for $i$ and $j$ positive integers, there exists $\left(Y_{i}^{*}, Y_{j}^{*}\right)$ distributed as $\left(Y_{i}, Y_{j}\right)$ and independent of $Y_{0}$ such that

$$
\frac{1}{2}\left\|\mathbb{E}\left(\mid Y_{i}-Y_{i}^{*} \| Y_{0}\right)+\mathbb{E}\left(\mid Y_{j}-Y_{j}^{*} \| Y_{0}\right)\right\|_{\infty}=\sup _{h \in \Lambda_{1}\left(\mathbb{R}^{2}\right)}\left\|\mathbb{E}\left(h\left(Y_{i}, Y_{j}\right) \mid Y_{0}\right)-\mathbb{E}\left(h\left(Y_{i}, Y_{j}\right)\right)\right\|_{\infty} .
$$

Notice now that for $i \geq j \geq 0$, by using the second part of Condition (3.4),

$$
\sup _{h \in \Lambda_{1}\left(\mathbb{R}^{2}\right)}\left\|\mathbb{E}\left(h\left(Y_{i}, Y_{j}\right) \mid Y_{0}\right)-\mathbb{E}\left(h\left(Y_{i}, Y_{j}\right)\right)\right\|_{\infty}=\sup _{h \in \Lambda_{1}\left(\mathbb{R}^{2}\right)}\left\|K^{j} \circ Q_{i-j}(h)-\nu\left(Q_{i-j}(h)\right)\right\|_{\infty, \nu} \leq C \rho^{j} .
$$


On an other hand, we clearly have that

$$
\begin{aligned}
\left\|K^{j}\left(f K^{i-j}(f)\right)-\nu\left(f K^{i-j}(f)\right)\right\|_{\infty, \nu} & =\left\|\mathbb{E}\left(f\left(Y_{i}\right) f\left(Y_{j}\right)-f\left(Y_{i}^{*}\right) f\left(Y_{j}^{*}\right) \mid Y_{0}\right)\right\|_{\infty} \\
& =\left\|\mathbb{E}\left(\left(f\left(Y_{i}\right)-f\left(Y_{i}^{*}\right)\right) f\left(Y_{j}\right) \mid Y_{0}\right)-\mathbb{E}\left(f\left(Y_{i}^{*}\right)\left(f\left(Y_{j}^{*}\right)-f\left(Y_{j}\right)\right) \mid Y_{0}\right)\right\|_{\infty} .
\end{aligned}
$$

Since $f$ is continuous on a compact set, there exists a positive constant $R$ such that $\|f\|_{\infty} \leq R$. Hence,

$$
\left\|K^{j}\left(f K^{i-j}(f)\right)-\nu\left(f K^{i-j}(f)\right)\right\|_{\infty, \nu} \leq R \| \mathbb{E}\left(( c ( | Y _ { i } - Y _ { i } ^ { * } | ) | Y _ { 0 } ) \| _ { \infty } + R \| \mathbb { E } \left(\left(c\left(\left|Y_{j}-Y_{j}^{*}\right|\right) \mid Y_{0}\right) \|_{\infty} .\right.\right.
$$

Since $c$ is concave and and non-decreasing function, it follows that

$$
\begin{aligned}
\left\|K^{j}\left(f K^{i-j}(f)\right)-\nu\left(f K^{i-j}(f)\right)\right\|_{\infty, \nu} & \leq R\left\|c\left(\mathbb{E}\left(\mid Y_{i}-Y_{i}^{*} \| Y_{0}\right)\right)\right\|_{\infty}+R\left\|c\left(\mathbb{E}\left(\mid Y_{j}-Y_{j}^{*} \| Y_{0}\right)\right)\right\|_{\infty} \\
& \leq R c\left(\left\|\mathbb{E}\left(\mid Y_{i}-Y_{i}^{*} \| Y_{0}\right)\right\|_{\infty}\right)+R c\left(\left\|\mathbb{E}\left(\mid Y_{j}-Y_{j}^{*} \| Y_{0}\right)\right\|_{\infty}\right) \\
& \leq 2 R c\left(\frac{1}{2}\left\|\mathbb{E}\left(\mid Y_{i}-Y_{i}^{*} \| Y_{0}\right)\right\|_{\infty}+\frac{1}{2}\left\|\mathbb{E}\left(\mid Y_{j}-Y_{j}^{*} \| Y_{0}\right)\right\|_{\infty}\right) .
\end{aligned}
$$

So overall,

$$
\sup _{i \geq j \geq n}\left\|K^{j}\left(f K^{i-j}(f)\right)-\nu\left(f K^{i-j}(f)\right)\right\|_{\infty, \nu} \leq 2 R c\left(C \rho^{n}\right)
$$

implying that (3.3) is satisfied with $\gamma=1 / \sqrt{3}$ as soon as (3.5) is. This ends the proof of the theorem.

\section{Proofs of the reverse martingale's results}

We start by recalling the following estimate of Hanson and Russo [12, Theorem 3.2A]

Lemma 6.1 Let $\left(B_{t}\right)_{t \geq 0}$ be a standard Brownian motion. Then

$$
\lim _{a \rightarrow \infty} \sup _{t \geq 0} \sup _{0 \leq s \leq a} \frac{\left|B_{t+s}-B_{t}\right|}{(2 a[\log ((t+a) / a))+\log \log a])^{1 / 2}}=1 \quad \mathbb{P} \text {-a.s. }
$$

We also recall the following convergence result for reverse martingales.

Lemma 6.2 Let $\left(\xi_{n}\right)_{n \geq 1}$ be a sequence of variables in $\mathbb{L}^{p}, 1 \leq p \leq 2$, adapted to a non-increasing filtration $\left(\mathcal{G}_{n}\right)_{n \geq 1}$. Assume that $\mathbb{E}\left(\xi_{n} \mid \mathcal{G}_{n+1}\right)=0$ and $\sum_{n \geq 1} \mathbb{E}\left(\left|\xi_{n}\right|^{p}\right)<\infty$. Then $\sum_{n \geq 1} \xi_{n}$ converges $\mathbb{P}$-a.s.and in $\mathbb{L}^{p}$.

Proof of Lemma 6.2. The result is clear when $p=1$, hence we assume $p>1$. Notice that for every $n>1,\left(\sum_{k=n-l}^{n} \xi_{k}\right)_{0 \leq l \leq n-1}$ is a $\left(\mathcal{G}_{n-l}\right)_{0 \leq l \leq n-1}$-martingale. Hence, by Burkholder inequality and using that $x \mapsto|x|^{p / 2}$ is subadditive, it follows that the exists a positive constant $C_{p}$ such that, for every $1 \leq r<n$,

$$
\mathbb{E}\left(\max _{r \leq m \leq n}\left|\sum_{k=m}^{n} \xi_{k}\right|^{p}\right) \leq C_{p} \mathbb{E}\left(\left(\sum_{k=r}^{n} \xi_{k}^{2}\right)^{p / 2}\right) \leq C_{p} \sum_{k=r}^{n} \mathbb{E}\left(\left|\xi_{k}\right|^{p}\right) .
$$

So, $\left(Z_{n}\right):=\left(\sum_{k=1}^{n} \xi_{k}\right)$ is Cauchy in $\mathbb{L}^{p}$, hence converges in $\mathbb{L}^{p}$, say to $Z$. Moreover, letting $n \rightarrow \infty$ in (6.2), we see that for every $r \geq 1$

$$
\mathbb{E}\left(\max _{m \geq r}\left|Z_{m}-Z\right|^{p}\right) \leq C_{p} \sum_{k \geq r} \mathbb{E}\left(\left|\xi_{k}\right|^{p}\right)
$$

which implies the desired result.

\subsection{Proof of Proposition 2.1.}

The $\mathbb{L}^{2}$ and a.s. convergence of $\sum_{k>1} \xi_{k}$ follows from Lemma 6.2. By Theorem 2 of Scott and Huggins [25], enlarging our probability space if necessary, there exists a Brownian motion $\left(B_{t}\right)_{t \geq 0}$, a non-increasing filtration $\left(\mathcal{H}_{n}\right)_{n \in \mathbb{N}}$ and a non-increasing process $\left(\tau_{n}\right)_{n \in \mathbb{N}}$ adapted to $\left(\mathcal{H}_{n}\right)_{n \in \mathbb{N}}$, such that

$$
R_{n}=B_{\tau_{n}} \quad \mathbb{P} \text {-a.s. }
$$


Moreover, writing $t_{n}:=\tau_{n}-\tau_{n+1} \geq 0 \mathbb{P}$-a.s., we have

$$
\begin{gathered}
\mathbb{E}\left(t_{n} \mid \mathcal{H}_{n+1}\right)=\mathbb{E}\left(\xi_{n}^{2} \mid \mathcal{G}_{n+1}\right) \quad \mathbb{P} \text {-a.s. } \\
\mathbb{E}\left(t_{n}^{p / 2} \mid \mathcal{H}_{n+1}\right) \leq C_{p} \mathbb{E}\left(\left|\xi_{n}\right|^{p} \mid \mathcal{G}_{n+1}\right) \quad \mathbb{P} \text {-a.s. } \quad \text { for every } p>1 .
\end{gathered}
$$

Hence, using (6.3) twice,

$$
\tau_{n}-\mathbb{E}\left(\tau_{n}\right)=\tau_{n}-\delta_{n}^{2}=\sum_{k \geq n}\left(t_{k}-\mathbb{E}\left(t_{k} \mid \mathcal{H}_{k+1}\right)\right)+V_{n}^{2}-\delta_{n}^{2} \quad \mathbb{P} \text {-a.s. }
$$

But it follows from (2.2) and (6.4) that $\sum_{n>1} \alpha_{n}^{-\nu} \mathbb{E}\left(t_{n}^{\nu}\right)<\infty$ which implies, by Lemma 6.2, that $\sum_{k \geq 1} \alpha_{k}^{-1}\left(t_{k}-\mathbb{E}\left(t_{k} \mid \mathcal{H}_{k+1}\right)\right)$ converges $\mathbb{P}$-a.s.. Then, by an analogue to the Kronecker lemma (see e.g. Heyde [14, Lemma 1] $), \sum_{k \geq n}\left(t_{k}-\mathbb{E}\left(t_{k} \mid \mathcal{H}_{k+1}\right)\right)=o\left(\alpha_{n}\right) \mathbb{P}$-a.s. Together with $(2.1)$, this implies in particular that $\tau_{n}-\delta_{n}^{2}=o\left(\alpha_{n}\right) \mathbb{P}$-a.s.

For every $t>0$ define $\widetilde{B}_{t}=t B_{1 / t}$, and $\widetilde{B}_{0}=0$. It is well-known that $\left(\widetilde{B}_{t}\right)_{t \geq 0}$ is a standard Brownian motion. We have $B_{\tau_{n}}-B_{\delta_{n}^{2}}=\tau_{n}\left(\widetilde{B}_{1 / \tau_{n}}-\widetilde{B}_{1 / \delta_{n}^{2}}\right)+\left(\tau_{n}-\delta_{n}^{2}\right) \widetilde{B}_{1 / \delta_{n}^{2}}$. By the law of the iterated logarithm for $\left(\widetilde{B}_{t}\right)_{t \geq 0}$ (or using that the supremum in (6.1) is greater than what we have for $t=0$ and $s=$ $\left.1 / \delta_{n}^{2}\right)$, we see that $\widetilde{B}_{1 / \delta_{n}^{2}}=O\left(\delta_{n}^{-1}\left(\log \log \left(1 / \delta_{n}\right)\right)^{1 / 2}\right) \mathbb{P}$-a.s.Hence since $\alpha_{n}=O\left(\delta_{n}^{2}\right),\left(\tau_{n}-\delta_{n}^{2}\right) \widetilde{B}_{1 / \delta_{n}^{2}}=$ $o\left(\left(\alpha_{n} \log \log \left(1 / \alpha_{n}\right)\right)^{1 / 2}\right) \mathbb{P}$-a.s.

Let us deal now with $\tau_{n}\left(\widetilde{B}_{1 / \tau_{n}}-\widetilde{B}_{1 / \delta_{n}^{2}}\right)$. With this aim, we shall use (6.1). Since $\alpha_{n}=O\left(\delta_{n}^{2}\right)$ and $\tau_{n}-\delta_{n}^{2}=o\left(\alpha_{n}\right) \mathbb{P}$-a.s., we have $\left|1 / \tau_{n}-1 / \delta_{n}^{2}\right|=o\left(1 / \alpha_{n}\right) \mathbb{P}$-a.s. Define $u_{n}:=\alpha_{n} /\left(\tau_{n} \delta_{n}^{2}\right), \varepsilon_{n}:=\max \left(\mid \delta_{n}^{2}-\right.$ $\left.\tau_{n} \mid / \alpha_{n}, u_{n}^{-1 / 2}\right), s_{n}:=\left|1 / \tau_{n}-1 / \delta_{n}^{2}\right|, a_{n}:=\varepsilon_{n} u_{n}$ and $v_{n}:=\min \left(1 / \delta_{n}^{2}, 1 / \tau_{n}\right)$. Notice that $a_{n} \rightarrow \infty, \varepsilon_{n} \rightarrow 0$, $v_{n}+s_{n}=\max \left(1 / \delta_{n}^{2}, 1 / \tau_{n}\right)$ and $\left|\widetilde{B}_{1 / \tau_{n}}-\widetilde{B}_{1 / \delta_{n}^{2}}\right|=\left|\widetilde{B}_{v_{n}+s_{n}}-\widetilde{B}_{v_{n}}\right|$. By (6.1), we have

$$
\leq \sup _{t \geq 0} \sup _{0 \leq s \leq a_{n}} \frac{\left|\widetilde{B}_{v_{n}+s_{n}}-\widetilde{B}_{v_{n}}\right|}{\left(2 a_{n}\left[\log \left(\left(v_{n}+a_{n}\right) / a_{n}\right)+\log \log a_{n}\right]\right)^{1 / 2}} \quad \frac{\left|\widetilde{B}_{t+s}-\widetilde{B}_{t}\right|}{\left.\left.\left(\log \left(\left(t+a_{n}\right) / a_{n}\right)\right)+\log \log a_{n}\right]\right)^{1 / 2}} \rightarrow 1 \quad \mathbb{P} \text {-a.s. }
$$

In particular, we have $\left|\widetilde{B}_{1 / \tau_{n}}-\widetilde{B}_{1 / \delta_{n}^{2}}\right|=O\left(\left[\varepsilon_{n} u_{n}\left(\left|\log \left(\delta_{n}^{2} /\left(\alpha_{n} \varepsilon_{n}\right)\right)\right|+\log \log \left(u_{n} \varepsilon_{n}\right)\right)\right]^{1 / 2}\right) \mathbb{P}$-a.s. Then, using that $\left|\log \left(\delta_{n}^{2} /\left(\alpha_{n} \varepsilon_{n}\right)\right)\right| \leq\left|\log \left(\delta_{n}^{2} / \alpha_{n}\right)\right|+\left|\log \varepsilon_{n}\right|$ and that $\varepsilon_{n} u_{n} \log \log \left(\varepsilon_{n} u_{n}\right)=o\left(u_{n} \log \log u_{n}\right)$, we obtain

$$
\tau_{n}\left(\widetilde{B}_{1 / \tau_{n}}-\widetilde{B}_{1 / \delta_{n}^{2}}\right)=o\left(\left[\alpha_{n}\left(\left|\log \left(\delta_{n}^{2} / \alpha_{n}\right)\right|+\log \log \left(\alpha_{n} / \delta_{n}^{4}\right)\right]^{1 / 2}\right) \quad \mathbb{P} \text {-a.s. },\right.
$$

which proves the result, since $1 / \delta_{n}^{4}=O\left(1 / \alpha_{n}^{2}\right)$.

Remark 6.3 It follows from the proof that the assumption $(2.2)$ may be replaced by $\sum_{k \geq n}\left(t_{k}-\mathbb{E}\left(t_{k} \mid \mathcal{H}_{k+1}\right)\right)=$ $o\left(\alpha_{n}\right) \mathbb{P}$-a.s.

\subsection{Proof of Theorem 2.3.}

Define $\xi_{n}:=X_{n} / \sigma_{n}^{2}$. Then, since $\mathbb{E}\left(\xi_{k}^{2}\right)=\left(\sigma_{k}^{2}-\sigma_{k-1}^{2}\right) \sigma_{k}^{-4}$, by comparing sums and integrals, it follows that $\sum_{k \geq 1} \mathbb{E}\left(\xi_{k}^{2}\right)<\infty$. Using the notations $V_{n}^{2}=\sum_{k \geq n}\left(\mathbb{E}\left(\xi_{k}^{2} \mid \mathcal{G}_{k+1}\right)\right.$ and $\delta_{n}^{2}=\sum_{k \geq n} \mathbb{E}\left(\xi_{k}^{2}\right)$, and writing $T_{n}:=\sum_{k=1}^{n}\left(\mathbb{E}\left(X_{k}^{2} \mid \mathcal{G}_{k+1}\right)-\mathbb{E}\left(X_{k}^{2}\right)\right)$, we have

$$
V_{n}^{2}-\delta_{n}^{2}=\sum_{k \geq n} \frac{T_{k}-T_{k-1}}{\sigma_{k}^{4}}=\sum_{k \geq n} T_{k}\left(\frac{1}{\sigma_{k}^{4}}-\frac{1}{\sigma_{k+1}^{4}}\right)-\frac{T_{n-1}}{\sigma_{n}^{4}} .
$$

Using (2.4) and that $\left(\sigma_{n}\right)_{n \in \mathbb{N}}$ and $\left(a_{n} / \sigma_{n}^{2}\right)_{n \in \mathbb{N}}$ are respectively non-decreasing and non-increasing, we obtain

$$
\left|V_{n}^{2}-\delta_{n}^{2}\right|=o\left(\frac{a_{n}}{\sigma_{n}^{2}}\right) \sum_{k \geq n}\left(\frac{1}{\sigma_{k}^{2}}-\frac{1}{\sigma_{k+1}^{2}}\right)+o\left(\frac{a_{n}}{\sigma_{n}^{4}}\right)=o\left(\frac{a_{n}}{\sigma_{n}^{4}}\right) .
$$

We want to apply Proposition 2.1 to $\left(\xi_{n}\right)$ with $\alpha_{n}:=a_{n} / \sigma_{n}^{4}$. Using $(2.5)$, we have

$$
\sum_{i \geq 1} \alpha_{i}^{-\nu} \mathbb{E}\left(\left|\xi_{i}\right|^{2 \nu}\right)=\sum_{n \geq 1} a_{i}^{-\nu} \mathbb{E}\left(\left|X_{i}\right|^{2 \nu}\right)<\infty
$$


hence condition (2.2) holds. It remains to prove that $\alpha_{n}=O\left(\delta_{n}^{2}\right)$ and that $\alpha_{n} / \delta_{n}^{4} \rightarrow \infty$. With this aim, we first notice that

$$
\frac{1}{\sigma_{n-1}^{2}}-\delta_{n}^{2}=\sum_{k \geq n} \int_{\sigma_{k-1}^{2}}^{\sigma_{k}^{2}}\left(\frac{1}{x^{2}}-\frac{1}{\sigma_{k}^{4}}\right) d x
$$

Hence, using that $\sup _{n} \mathbb{E}\left(X_{n}^{2}\right)<\infty$, it follows that $\sigma_{n}=O\left(\sigma_{n-1}\right)$ and

$$
0 \leq \frac{1}{\sigma_{n-1}^{2}}-\delta_{n}^{2} \leq \sum_{k \geq n} \mathbb{E}\left(X_{k}^{2}\right)\left(\frac{1}{\sigma_{k-1}^{4}}-\frac{1}{\sigma_{k}^{4}}\right)=O\left(\sum_{k \geq n} \frac{\sigma_{k}^{2}-\sigma_{k-1}^{2}}{\sigma_{k}^{6}}\right)=O\left(\frac{\delta_{n}^{2}}{\sigma_{n}^{2}}\right) .
$$

In particular, since $\delta_{n}^{2}=O\left(\sigma_{n-1}^{-2}\right)=O\left(\sigma_{n}^{-2}\right)$ and $\left|\sigma_{n}^{-2}-\sigma_{n-1}^{-2}\right|=O\left(\sigma_{n}^{-4}\right)$, we have

$$
\left|\frac{1}{\sigma_{n}^{2}}-\delta_{n}^{2}\right|=O\left(\frac{1}{\sigma_{n}^{4}}\right)
$$

Since $a_{n} \sigma_{n}^{-2}$ is non-increasing, (6.6) implies that $\alpha_{n}=O\left(\delta_{n}^{2}\right)$. In addition since $a_{n}$ is tending to infinity, (6.6) entails also that $\alpha_{n} / \delta_{n}^{4} \rightarrow \infty$.

By Proposition 2.1, enlarging our probability space if necessary, there exists a standard Brownian motion $\left(B_{t}\right)_{t \geq 0}$, such that $(2.3)$ holds with $\delta_{n}^{2}=\sum_{k \geq n}\left(\sigma_{k}^{2}-\sigma_{k-1}^{2}\right) \sigma_{k}^{-4}$. Now, for every $t>0$ define $\widetilde{B}_{t}=t B_{1 / t}$, and $\widetilde{B}_{0}=0$ (recall that $\left(\widetilde{B}_{t}\right)_{t \geq 0}$ is a standard Brownian motion). Notice that

$$
B_{1 / \sigma_{n}^{2}}-B_{\delta_{n}^{2}}=\sigma_{n}^{-2}\left(\widetilde{B}_{\sigma_{n}^{2}}-\widetilde{B}_{1 / \delta_{n}^{2}}\right)+\left(\sigma_{n}^{-2}-\delta_{n}^{2}\right) \widetilde{B}_{1 / \delta_{n}^{2}}
$$

By (6.6) and the law of the iterated logarithm for $\left(\widetilde{B}_{t}\right)_{t \geq 0}$, we derive that

$$
\left(\sigma_{n}^{-2}-\delta_{n}^{2}\right) \widetilde{B}_{1 / \delta_{n}^{2}}=O\left(\sigma_{n}^{-2}\left(\log \log \left(\sigma_{n}\right)\right)^{1 / 2}\right)=o\left(\frac{\left(a_{n}\left(\log \log a_{n}\right)\right)^{1 / 2}}{\sigma_{n}^{2}}\right) \mathbb{P} \text {-a.s. }
$$

To deal now with $\sigma_{n}^{-2}\left(\widetilde{B}_{\sigma_{n}^{2}}-\widetilde{B}_{1 / \delta_{n}^{2}}\right)$, we use the same arguments as the ones used to derive (6.5) (with $\sigma_{n}^{-2}$ replacing $\left.\tau_{n}\right)$. Hence we infer that

$$
\sigma_{n}^{-2}\left(\widetilde{B}_{\sigma_{n}^{2}}-\widetilde{B}_{1 / \delta_{n}^{2}}\right)=o\left(\frac{\left(a_{n}\left(\left|\log \left(\sigma_{n}^{2} / a_{n}\right)\right|+\log \log a_{n}\right)\right)^{1 / 2}}{\sigma_{n}^{2}}\right) \quad \mathbb{P} \text {-a.s. }
$$

So, overall, it follows that

$$
\left|R_{n}-B_{1 / \sigma_{n}^{2}}\right|=o\left(\frac{\left(a_{n}\left(\left|\log \left(\sigma_{n}^{2} / a_{n}\right)\right|+\log \log a_{n}\right)\right)^{1 / 2}}{\sigma_{n}^{2}}\right) \quad \mathbb{P} \text {-a.s. }
$$

where $R_{n}=\sum_{k \geq n} X_{n} / \sigma_{n}^{2}$.

Write $\widetilde{Z}_{n}:=\sigma_{n}^{2}\left(B_{1 / \sigma_{n}^{2}}-B_{1 / \sigma_{n+1}^{2}}\right)$. By independence of the increments, $\left(\widetilde{Z}_{n}\right)$ is a sequence of independent centered Gaussian variables. Notice that, by stationarity of the increments $\mathbb{E}\left(\widetilde{Z}_{n}^{2}\right)=\sigma_{n}^{2} \mathbb{E}\left(X_{n}^{2}\right) / \sigma_{n+1}^{2}$.

We have

$$
\begin{aligned}
\sum_{k=1}^{n} X_{k}-\sum_{k=1}^{n} \widetilde{Z}_{k} & =\sum_{k=1}^{n} \sigma_{k}^{2}\left(\left(R_{k}-B_{1 / \sigma_{k}^{2}}\right)-\left(R_{k+1}-B_{1 / \sigma_{k+1}^{2}}\right)\right) \\
& =\sum_{k=2}^{n}\left(R_{k}-B_{1 / \sigma_{k}^{2}}\right)\left(\sigma_{k}^{2}-\sigma_{k-1}^{2}\right)+\sigma_{1}^{2}\left(R_{1}-B_{1 / \sigma_{1}^{2}}\right)-\sigma_{n}^{2}\left(R_{n+1}-B_{1 / \sigma_{n+1}^{2}}\right) .
\end{aligned}
$$

Using that $\left(\sigma_{n}\right),\left(\sigma_{n}^{2} / a_{n}\right),\left(a_{n} / \sigma_{n}\right)$ and $\left(a_{n}\right)$ are non-decreasing, and taking into account $(6.7)$, we deduce that

$$
\begin{aligned}
\sum_{k=1}^{n} X_{k}-\sum_{k=1}^{n} \widetilde{Z}_{k} & =o\left(\left(\frac{a_{n}\left(\left|\log \left(\sigma_{n}^{2} / a_{n}\right)\right|+\log \log a_{n}\right)}{\sigma_{n}}\right)^{1 / 2}\right) \sum_{k=2}^{n} \frac{\sigma_{k}^{2}-\sigma_{k-1}^{2}}{\left(\sigma_{k}^{2}\right)^{3 / 4}} \\
& =o\left(\left(a_{n}\left(\left|\log \left(\sigma_{n}^{2} / a_{n}\right)\right|+\log \log a_{n}\right)^{1 / 2}\right),\right.
\end{aligned}
$$


where we used that $\sum_{k=2}^{n}\left(\sigma_{k}^{2}-\sigma_{k-1}^{2}\right)\left(\sigma_{k}^{2}\right)^{-3 / 4}=O\left(\int_{0}^{\sigma_{n}^{2}} d x / x^{3 / 4}\right)$.

Finally, define $Z_{n}:=\widetilde{Z}_{n} \sigma_{n+1} / \sigma_{n}$. Notice that $\left|Z_{n}-\widetilde{Z}_{n}\right| \leq C\left|\widetilde{Z}_{n}\right| / \sigma_{n}$ for some $C>0$. Hence $\sum_{n \geq 1} \mathbb{E}\left(\left(Z_{n}-\widetilde{Z}_{n}\right)^{2}\right) / a_{n} \leq \sum_{n \geq 1}\left(\sigma_{n}^{2}-\sigma_{n-1}^{2}\right) / \sigma_{n}^{3}<\infty$. So, by the Kolmogorov theorem (see also Lemma $6.2), \sum_{n \geq 1}\left(Z_{n}-\widetilde{Z}_{n}\right) / \sqrt{a_{n}}$ converges $\mathbb{P}$-a.s., and (2.6) follows from the Kronecker lemma.

\subsection{Proof of Corollary 2.5.}

Assume that $\mathbb{E}\left(X_{1}^{2}\right) \neq 0$, otherwise there is nothing to prove. We start by the proof of Corollary 2.5 . Notice first that by stationarity and Fubini theorem,

$$
\sum_{n \geq 1} \frac{\mathbb{E}\left(\left|X_{n}\right| \mathbf{1}_{\left\{\left|X_{n}\right|>\sqrt{n}\right\}}\right)}{\sqrt{n}}=\mathbb{E}\left(\left|X_{1}\right| \sum_{1 \leq n<X_{1}^{2}} \frac{1}{\sqrt{n}}\right) \leq C \mathbb{E}\left(X_{1}^{2}\right)<\infty .
$$

Hence,

$$
\sum_{n \geq 1} n^{-1 / 2}\left|X_{n}\right| \mathbf{1}_{\left\{\left|X_{n}\right|>\sqrt{n}\right\}}<\infty \quad \mathbb{P} \text {-a.s. and } \sum_{n \geq 1} n^{-1 / 2} \mathbb{E}\left(\left|X_{n}\right| \mathbf{1}_{\left\{\left|X_{n}\right|>\sqrt{n}\right\}} \mid \mathcal{G}_{n+1}\right)<\infty \quad \mathbb{P} \text {-a.s. }
$$

and by the Kronecker lemma,

$$
\sum_{k=1}^{n}\left|X_{k}\right| \mathbf{1}_{\left\{\left|X_{k}\right|>\sqrt{k}\right\}}=o(\sqrt{n}) \quad \mathbb{P} \text {-a.s. and } \sum_{k=1}^{n} \mathbb{E}\left(\left|X_{k}\right| \mathbf{1}_{\left\{\left|X_{k}\right|>\sqrt{k}\right\}} \mid \mathcal{G}_{k+1}\right)=o(\sqrt{n}) \quad \mathbb{P} \text {-a.s. }
$$

Define $Y_{n}:=X_{n} \mathbf{1}_{\left\{\left|X_{n}\right| \leq \sqrt{n}\right\}}-\mathbb{E}\left(X_{n} \mathbf{1}_{\left\{\left|X_{n}\right| \leq \sqrt{n}\right\}} \mid \mathcal{G}_{n+1}\right)$. Then, by the above, using that $\mathbb{E}\left(X_{n} \mid \mathcal{G}_{n+1}\right)=0$ a.s., we see that it suffices to prove $(2.7)$ with $\left(X_{n}\right)$ replaced with $\left(Y_{n}\right)$.

We want to apply Theorem 2.3 to $\left(Y_{n}\right)$ with $a_{n}=\sigma_{n}^{2}=n$. We have to prove conditions (2.4) and (2.5). Let us prove (2.4). Clearly, $\left(\mathbb{E}\left(Y_{1}^{2}\right)+\cdots+\mathbb{E}\left(Y_{n}^{2}\right)\right) / n \rightarrow \mathbb{E}\left(X_{1}^{2}\right)$. Hence, we only need to prove that

$$
\left(\mathbb{E}\left(Y_{1}^{2} \mid \mathcal{G}_{2}\right)+\cdots+\mathbb{E}\left(Y_{n}^{2} \mid \mathcal{G}_{n+1}\right)\right) / n \rightarrow \mathbb{E}\left(X_{1}^{2}\right) \quad \mathbb{P} \text {-a.s. }
$$

We first prove that

$$
\left(\mathbb{E}\left(Y_{1}^{2} \mid \mathcal{G}_{2}\right)+\cdots+\mathbb{E}\left(Y_{n}^{2} \mid \mathcal{G}_{n+1}\right)\right)-\left(Y_{1}^{2}+\cdots+Y_{n}^{2}\right)=o(n) \quad \mathbb{P} \text {-a.s. }
$$

By Kronecker lemma, this will follow from the convergence of the series $\sum_{n}\left(\mathbb{E}\left(Y_{n}^{2} \mid \mathcal{G}_{n+1}\right)-Y_{n}^{2}\right) / n$. By Lemma 6.2, this last convergence will hold true provided that $\sum_{n} \mathbb{E}\left(Y_{n}^{4}\right) / n^{2}<\infty$. But, by stationarity and Fubini theorem, we have

$$
\sum_{n \geq 1} \frac{\mathbb{E}\left(Y_{n}^{4}\right)}{n^{2}} \leq 16 \mathbb{E}\left(X_{1}^{4} \sum_{n \geq X_{1}^{2}} \frac{1}{n^{2}}\right) \leq C \mathbb{E}\left(X_{1}^{2}\right)<\infty .
$$

Therefore (6.11) is proved. Now, by the ergodic theorem we have

$$
\limsup _{n} \frac{\sum_{k=1}^{n} X_{k}^{2} \mathbf{1}_{\left\{\left|X_{k}\right| \leq \sqrt{k}\right\}}}{n} \leq \lim _{n} \frac{\sum_{k=1}^{n} X_{k}^{2}}{n}=\mathbb{E}\left(X_{1}^{2}\right) \quad \mathbb{P} \text {-a.s. },
$$

and for any $A$ fixed,

$$
\liminf _{n} \frac{\sum_{k=1}^{n} X_{k}^{2} \mathbf{1}_{\left\{\left|X_{k}\right| \leq \sqrt{k}\right\}}}{n} \geq \lim _{n} \frac{\sum_{k=1}^{n} X_{k}^{2} \mathbf{1}_{\left\{\left|X_{k}\right| \leq A\right\}}}{n}=\mathbb{E}\left(X_{1}^{2} \mathbf{1}_{\left\{\left|X_{1}\right| \leq A\right\}}\right) \quad \mathbb{P} \text {-a.s. }
$$

Letting $A \rightarrow \infty$, we see that the liminf and the $\lim \sup$ above are equal to $\mathbb{E}\left(X_{1}^{2}\right)$. Hence

$$
\frac{\sum_{k=1}^{n} X_{k}^{2} \mathbf{1}_{\left\{\left|X_{k}\right| \leq \sqrt{k}\right\}}}{n} \rightarrow \mathbb{E}\left(X_{1}^{2}\right) \quad \mathbb{P} \text {-a.s. }
$$

On an other hand using the fact that $\mathbb{E}\left(X_{k} \mid \mathcal{G}_{k+1}\right)=0$ a.s. together with (6.9), we get that

$$
n^{-1} \sum_{k=1}^{n}\left(\mathbb{E}\left(X_{k} \mathbf{1}_{\left\{\left|X_{k}\right| \leq \sqrt{k}\right\}} \mid \mathcal{G}_{k+1}\right)\right)^{2} \leq n^{-1 / 2} \sum_{k=1}^{n} \mathbb{E}\left(\left|X_{k}\right| \mathbf{1}_{\left\{\left|X_{k}\right|>\sqrt{k}\right\}} \mid \mathcal{G}_{k+1}\right)=o(1) \quad \mathbb{P} \text {-a.s. }
$$


Combining (6.13), (6.14) and (6.11), we see that (6.10) holds, which proves (2.4).

The fact that (2.5) holds with $\nu=2$ follows from (6.12). By Theorem 2.3, there exists a sequence of independent centered Gaussian variables $\left(\widetilde{Z}_{n}\right)_{n \geq 1}$ such that $\mathbb{E}\left(\widetilde{Z}_{n}^{2}\right)=\mathbb{E}\left(Y_{n}^{2}\right)=\mathbb{E}\left(X_{1}^{2}\right)+o(1)$ and $Y_{1}+\cdots Y_{n}-\left(\widetilde{Z}_{1}+\cdots+\widetilde{Z}_{n}\right)=o(\sqrt{n \log \log n}) \mathbb{P}$-a.s. Let $\left(\delta_{k}\right)_{k \geq 1}$ be a sequence of iid Gaussian random variables with mean zero and variance $\mathbb{E}\left(X_{1}^{2}\right)$, independent of the sequence $\left(\widetilde{Z}_{n}\right)_{n \geq 1}$. We now construct a sequence $\left(Z_{n}\right)_{n \geq 1}$ as follows. If $\mathbb{E}\left(\widetilde{Z}_{n}^{2}\right)=0$, then $Z_{n}=\delta_{n}$, else $Z_{n}=c_{n} \widetilde{Z}_{n}$ where $c_{n}=\sqrt{\frac{\mathbb{E}\left(X_{1}^{2}\right)}{\mathbb{E}\left(\widetilde{Z}_{n}^{2}\right)}}$. By construction, the $Z_{n}$ 's are iid Gaussian random variables with mean zero and variance $\mathbb{E}\left(X_{1}^{2}\right)$. Write $G_{n}:=Z_{n}-\widetilde{Z}_{n}$ and $v_{n}^{2}:=\sum_{k=1}^{n} \mathbb{E}\left(G_{k}^{2}\right)$. By Lévy's inequality (see for instance Proposition 2.3 in [16]),

$$
\mathbb{P}\left(\max _{1 \leq k \leq 2^{r}}\left|\sum_{i=1}^{k} G_{i}\right|>x\right) \leq 2 \exp \left(-\frac{x^{2}}{2 v_{2^{r}}^{2}}\right) .
$$

Hence taking $x=2 v_{2^{r}}\left(\log \log 2^{r}\right)^{1 / 2}$, we get that

$$
\sum_{r \geq 0} \mathbb{P}\left(\max _{1 \leq k \leq 2^{r}}\left|\sum_{i=1}^{k} G_{i}\right|>2 v_{2^{r}}\left(\log \log 2^{r}\right)^{1 / 2}\right)<\infty .
$$

Therefore $\sup _{1 \leq k \leq 2^{r}}\left|\sum_{i=1}^{k} G_{i}\right|=O\left(v_{2^{r}}\left(\log \log 2^{r}\right)^{1 / 2}\right)$ almost surely. $\mathbb{P}$-a.s. This ends the proof of Corollary 2.5 since $v_{n}^{2}=o(n)$.

\subsection{Proof of Corollary 2.7.}

Define $Y_{n}:=X_{n} \mathbf{1}_{\left\{\left|X_{n}\right| \leq n^{1 / p}\right\}}-\mathbb{E}\left(X_{n} \mathbf{1}_{\left\{\left|X_{n}\right| \leq n^{1 / p}\right\}} \mid \mathcal{G}_{n+1}\right)$. Since $\mathbb{E}\left(X_{n} \mid \mathcal{G}_{n+1}\right)=0$ a.s.,

$$
\sum_{k \geq 1} \frac{\mathbb{E}\left|X_{k}-Y_{k}\right|}{k^{1 / p}} \leq 2 \sum_{k \geq 1} \frac{\mathbb{E}\left(\left|X_{k}\right| \mathbf{1}_{\left\{\left|X_{n}\right| \leq k^{1 / p}\right\}}\right)}{k^{1 / p}} .
$$

Hence by stationary and Fubini theorem, $\sum_{k \geq 1} k^{-1 / p} \mathbb{E}\left|X_{k}-Y_{k}\right|<\infty$, implying via the Kronecker lemma that

$$
\sum_{k=1}^{n}\left|X_{k}-Y_{k}\right|=o\left(n^{1 / p}\right) \quad \mathbb{P} \text {-a.s. }
$$

Let us prove now that $\left(Y_{n}\right)_{n \geq 1}$ satisfies the conditions of Theorem 2.3 with $a_{n}=n^{2 / p} b(n)$ and $\sigma_{n}^{2}=n$. With this aim, we first notice that since $\mathbb{E}\left(X_{n} \mid \mathcal{G}_{n+1}\right)=0$ a.s.,

$$
\mathbb{E}\left(X_{k}^{2} \mid \mathcal{G}_{k+1}\right)-\mathbb{E}\left(X_{k}^{2}\right)-\mathbb{E}\left(Y_{k}^{2} \mid \mathcal{G}_{k+1}\right)+\mathbb{E}\left(Y_{k}^{2}\right)=\mathbb{E}\left(X_{k}^{2} \mathbf{1}_{\left\{\left|X_{k}\right|>k^{1 / p}\right\}} \mid \mathcal{G}_{k+1}\right)-\mathbb{E}\left(X_{k}^{2} \mathbf{1}_{\left\{\left|X_{k}\right|>k^{1 / p}\right\}}\right) .
$$

Since by stationarity and Fubini theorem, $\sum_{k \geq 1} k^{-2 / p} \mathbb{E}\left(X_{k}^{2} \mathbf{1}_{\left\{\left|X_{k}\right|>k^{1 / p}\right\}}\right)<\infty$, we conclude via the Kronecker lemma that

$$
\sum_{k=1}^{n}\left|\mathbb{E}\left(X_{k}^{2} \mid \mathcal{G}_{k+1}\right)-\mathbb{E}\left(X_{k}^{2}\right)-\mathbb{E}\left(Y_{k}^{2} \mid \mathcal{G}_{k+1}\right)+\mathbb{E}\left(Y_{k}^{2}\right)\right|=o\left(n^{2 / p}\right) \quad \mathbb{P} \text {-a.s. }
$$

Together with condition (2.8), this implies that

$$
\sum_{k=1}^{n}\left(\mathbb{E}\left(Y_{k}^{2} \mid \mathcal{G}_{k+1}\right)-\mathbb{E}\left(Y_{k}^{2}\right)\right)=o\left(n^{2 / p} b(n)\right) \quad \mathbb{P} \text {-a.s. }
$$

Notice now that by stationarity and Fubini theorem,

$$
\sum_{n \geq 1} \frac{\mathbb{E}\left(Y_{n}^{4}\right)}{n^{4 / p}} \leq 16 \mathbb{E}\left(X_{1}^{4} \sum_{n \geq\left|X_{1}\right|^{p}} \frac{1}{n^{4 / p}}\right) \leq C_{p} \mathbb{E}\left(\left|X_{1}\right|^{p}\right)<\infty,
$$

Therefore $\left(Y_{n}\right)_{n \geq 1}$ satisfies (2.5) with $\nu=2$. Applying Theorem 2.3, we conclude that enlarging our probability space if necessary, there exists a sequence a sequence of independent centered Gaussian 
variables $\left(\widetilde{Z}_{n}\right)_{n \geq 1}$ such that $\mathbb{E}\left(\widetilde{Z}_{n}^{2}\right)=\mathbb{E}\left(Y_{n}^{2}\right)$ and $Y_{1}+\cdots Y_{n}-\left(\widetilde{Z}_{1}+\cdots+\widetilde{Z}_{n}\right)=o\left(n^{1 / p} \sqrt{b(n) \log n}\right) \mathbb{P}$-a.s. We consider now the sequence of iid centered Gaussian variables $\left(Z_{n}\right)_{n \geq 1}$ with variance $\mathbb{E}\left(X_{1}^{2}\right)$ as defined in the proof of Corollary 2.5. Notice then that

$$
\mathbb{E}\left(Z_{k}-\tilde{Z}_{k}\right)^{2}=\left(\left\|X_{k}\right\|_{2}-\left\|Y_{k}\right\|_{2}\right)^{2} \leq\left\|X_{k}-Y_{k}\right\|_{2}^{2} \leq \mathbb{E}\left(X_{k}^{2} \mathbf{1}_{\left\{\left|X_{k}\right|>k^{1 / p}\right\}}\right),
$$

where for the last inequality, we have used the fact that $\mathbb{E}\left(X_{n} \mid \mathcal{G}_{n+1}\right)=0$ a.s. Hence by stationarity

$$
\sum_{n \geq 1} \mathbb{E}\left(Z_{n}-\tilde{Z}_{n}\right)^{2} / n^{2 / p} \leq \mathbb{E}\left(X_{1}^{2} \sum_{1 \leq n \leq\left|X_{1}\right|^{p}} 1 / n^{2 / p}\right) \leq C \mathbb{E}\left(\left|X_{1}\right|^{p}\right)<\infty .
$$

Therefore by the Kolmogorov theorem (or Lemma 6.2), $\sum_{n>1}\left(Z_{n}-\tilde{Z}_{n}\right) / n^{2 / p}$ converges $\mathbb{P}$-a.s.and by the Kronecker lemma $Z_{1}+\ldots+Z_{n}-\left(\widetilde{Z}_{1}+\ldots+\widetilde{Z}_{n}\right)=o\left(n^{1 / p} \sqrt{\log \log n}\right) \mathbb{P}$-a.s. This achieves the proof of Corollary 2.7.

\subsection{Proof of Corollary 2.8.}

The proof relies more deeply on the construction of Scott and Huggins [25]. We want to use Theorem 2.3 without condition (2.5). Now the proof of Theorem 2.3 relies on Proposition 2.1 and (2.5) is used to ensure that condition (2.2) holds for an auxiliary process. Instead of (2.5) we will make use of Remark 6.3. We define a reverse martingale $\left(R_{n}\right)_{n \geq 1}$, by $R_{n}=\sum_{k \geq n} X_{k} / k$. Notice that $R_{n}$ is well defined in $\mathbb{L}^{2}$ by Lemma 6.2 .

For every $n \leq-1$ define $\widetilde{R}_{n}:=R_{-n}, \widetilde{X}_{n}:=X_{-n}$ and $\widetilde{\mathcal{G}}_{n}:=\mathcal{G}_{-n}$. Then $\left(\widetilde{R}_{n}, \widetilde{\mathcal{G}}_{n}\right)_{n \leq-1}$ is a martingale.

Enlarging our probability space if necessary, we may consider a countable set of standard Brownian motions $\left(B_{t}^{(n)}\right)_{t \geq 0}, n \leq-1$ that are independent of each others and of $\left(\widetilde{X}_{n}\right)_{n \leq-1}$. Notice that the process $\left(\widetilde{X}_{n},\left(B_{t}^{(n)}\right)_{t \geq 0}\right)_{n \leq-1}$ with values in $\mathbb{R} \times \mathbb{R}^{\mathbb{R}^{+}}$, is stationary.

We now define a filtration $\left(\widetilde{\mathcal{H}}_{t}\right)_{t \leq-1}$ as follows. For $n \leq-1$ an integer, write $\widetilde{\mathcal{H}}_{n}=\widetilde{\mathcal{G}}_{n} \vee \sigma\left\{B_{t}^{(j)}, 0 \leq\right.$ $t<\infty,-\infty<j \leq n\}$. For every $t \leq-1$, not an integer, write $\widetilde{\mathcal{H}}_{t}=\widetilde{\mathcal{H}}_{[t]} \vee\left\{R_{[t]+1}+B_{\phi(s)}^{([t]+1)}, 0<s \leq\right.$ $t-[t]\}$, where $[t]$ stands for the largest negative integer, not exceeding $t$, and $\phi$ is defined on $] 0,1]$ by $\phi(s):=1 / s-1$. Then, we define a continuous martingale with respect to $\left(\widetilde{\mathcal{H}}_{t}\right)_{t \leq-1}$ interpolating $\left(\widetilde{R}_{n}\right)$, by $\widetilde{R}_{t}=\mathbb{E}\left(\widetilde{R}_{[t]+1} \mid \widetilde{\mathcal{H}}_{t}\right)$, for every $t \leq-1$. Notice that

$$
\widetilde{R}_{t}=\widetilde{R}_{[t]}+\frac{\mathbb{E}\left(X_{-[t]-1} \mid \widetilde{\mathcal{H}}_{t}\right)}{-[t]-1} .
$$

Using Theorem A of [25] as done page 451 of [25], there exists a continuous non-decreasing process $\left(\widetilde{\tau}_{t}\right)_{t \leq-1}$ and a Brownian motion $\left(B_{t}^{*}\right)_{t \geq 0}$ such that $\widetilde{R}_{t}=B_{\widetilde{\tau}_{t}}^{*}$ a.s. and $\left(\widetilde{R}_{t}^{2}-\widetilde{\tau}_{t}\right)_{t \leq-1}$ is a martingale with respect to $\left(\widetilde{\mathcal{H}}_{t}\right)_{t \leq-1}$. In particular, $\left(\widetilde{\tau}_{t}\right)_{t \leq-1}$ must be the quadratic variation of $\left(\widetilde{R}_{t}\right)$ on $\left.]-\infty, t\right]$.

For every $n \geq 1$, define $\tau_{n}:=\widetilde{\tau}_{-n}, \mathcal{H}_{n}:=\widetilde{\mathcal{H}}_{-n}$. These are exactly the quantities involved in the proof of Proposition 2.1. Then $t_{n}=\tau_{n}-\tau_{n+1}$ is nothing else but the quadratic variation of $\left(\widetilde{R}_{t}\right)$ on $[-n-1,-n]$. But it follows from (6.16) that $\left(n^{2} t_{n}\right)_{n \geq 1}$ is a stationary and ergodic process.

By Remark 6.3 we need to prove

$$
\sum_{k \geq n}\left(t_{k}-\mathbb{E}\left(t_{k} \mid \mathcal{H}_{k+1}\right)\right)=O\left(n^{-3 / 2} \sqrt{\log \log n}\right) \quad \mathbb{P} \text {-a.s. }
$$

Since $\left(n^{2} t_{n}\right)_{n \geq 1}$ is a stationary and ergodic sequence in $\mathbb{L}^{2}$, it follows from Corollary 2.5 that $\sum_{k=1}^{n} k^{2}\left(t_{k}-\right.$ $\left.\mathbb{E}\left(t_{k} \mid \mathcal{H}_{k+1}\right)\right)=O(\sqrt{n \log \log n}) \mathbb{P}$-a.s., which proves (6.17) by an Abel summation.

\section{References}

[1] Broise, A. Transformations dilatantes de l'intervalle et théorèmes limites. Études spectrales d'opérateurs de transfert et applications. Astérisque 238 (1996), 1-109. 
[2] Collet, P., Martinez, S. and Schmitt, B. Exponential inequalities for dynamical measures of expanding maps of the interval. Probab. Theory. Relat. Fields. 123 (2002), 301-322.

[3] Cuny, C. Pointwise ergodic theorems with rate and application to limit theorems for stationary processes. Stoch. Dyn. 11 (2011), no. 1, 135-155.

[4] Dedecker, J., Doukhan, P. and Merlevède, F. Rates of convergence in the strong invariance principle under projective criteria. Electron. J. Probab. 17 (2012), no. 16, 1-31.

[5] Dedecker, J., Gouëzel, S. and Merlevède, F. Some almost sure results for intermittent maps and their associated Markov chains. Ann. Inst. H. Poincaré Probab. Statist. 46 (2010), 796-821.

[6] Dedecker, J., Gouëzel, S. and Merlevède F. The almost sure invariance principle for unbounded functions of expanding maps. ALEA, Lat. Am. J. Probab. Math. Stat. 9 (2012), 141-163.

[7] Dedecker, J., Merlevède, F., Peligrad, M. and Utev, S. Moderate deviations for stationary sequences of bounded random variables. Ann. Inst. Henri Poincaré Probab. Stat. 45 (2009), 453-476.

[8] Dedecker, J. and Prieur, C. An empirical central limit theorem for dependent sequences. Stochastic Process. Appl. 117 (2007), 121-142.

[9] Dedecker, J. and Prieur, C. New dependence coefficients. Examples and applications to statistics. Probab. Theory Related Fields. 132 (2005), 203-236.

[10] Field, M.J., Melbourne, I., Török, A. Decay of correlations, central limit theorems and approximation by Brownian motion for compact Lie group extensions. Ergod. Th. \& Dyn. Sys. 23 (2003), 87-110.

[11] Gouëzel, S. Almost sure invariance principle for dynamical systems by spectral methods. Ann. Probab. 38 (2010), no. 4, 1639-1671.

[12] Hanson, D.L. and Russo, R. P. Some results on increments of the Wiener process with applications to lag sums of i.i.d. random variables. Ann. Probab. 11 (1983), no. 3, 609-623.

[13] Hennion, H. and Hervé. L. Limit theorems for Markov chains and stochastic properties of dynamical systems by quasi-compactness. Lecture Notes in Mathematics 1766 Springer. (2001).

[14] Heyde, C.C. On central limit and iterated logarithm supplements to the martingale convergence theorem. J. Appl. Probability 14 (1977), no. 4, 758-75.

[15] Huggins, R.M. A strong invariance principle for reverse martingales. Acta Math. Hungar. 46 (1985), no. $1-2,93-100$.

[16] Ledoux, M. and Talagrand, M. Probability in Banach spaces. Isoperimetry and processes. SpringerVerlag, Berlin. 1991.

[17] Melbourne, I. and Nicol, M. Almost sure invariance principle for nonuniformly hyperbolic systems. Commun. Math. Phys. 260 (2005), 131-146.

[18] Melbourne, I. and Nicol, M. A vector-valued almost sure invariance principle for hyperbolic dynamical systems. Ann. Probab. (2009), 478-505.

[19] Merlevède, F., Peligrad, C. and Peligrad, M. (2012). Almost Sure Invariance Principles via Martingale Approximation. Stochastic Process. Appl. 122 (2012), 170-190.

[20] Merlevède, F. and Peligrad, M. Rosenthal inequalities for martingales and stationary sequences and examples. to appear in Ann. Probab. (2012). arXiv:1103.3242.

[21] Merlevède, F. and Rio, E. Strong approximation of partial sums under dependence conditions with application to dynamical systems. Stochastic Process. Appl. 122 (2012), 386-417.

[22] Peligrad, M. and Utev, S. A new maximal inequality and invariance principle for stationary sequences. Ann. Probab. 33 (2005), no. 2, 798-815.

[23] Peligrad, M. and Utev, S. Central limit theorem for stationary linear processes. Ann. Probab. 34 (2006), 1608-1622. 
[24] Philipp, W. and Stout, W.F. Almost sure invariance principle for partial sums of weakly dependent random variables. Mem. of the Amer. Math. Soc. 161 (1975), Providence, RI: Amer. Math. Soc.

[25] Scott, D.J. and Huggins, R.M. On the embedding ofprocesses in Brownian motion and the law of the iterated logarithm for reverse martingales. Bull. Austral. Math. Soc. 27 (1983), no. 3, 443-459

[26] Shao, Q.M. Almost sure invariance principles for mixing sequences of random variables. Stochastic Process. Appl. 48 (1993), 319-334.

[27] Strassen, V. Almost sure behavior of sums of independent random variables and martingales. Proc. Fifth Berkeley Sympos. Math. Statist. and Probability (Berkeley, Calif., 1965/66) II 315-343. Univ. California Press, Berkeley, Calif. (1967).

[28] Wu, L. Functional law of iterated logarithm for additive functionals of reversible Markov processes. Acta Math. Appl. Sinica, 16 (2000), no. 2, 149-161.

[29] Wu, W.B. Strong invariance principles for dependent random variables. Ann. Probab. 35 (2007), $2294-2320$. 